\title{
Holography in 3D AdS gravity with torsion
}

\author{
Milutin Blagojević, ${ }^{a}$ Branislav Cvetković, ${ }^{a}$ Olivera Miskovic ${ }^{b}$ and Rodrigo Olea. ${ }^{c}$ \\ ${ }^{a}$ Institute of Physics, University of Belgrade, \\ P. O. Box 57, 11001 Belgrade, Serbia \\ ${ }^{b}$ Instituto de Física, Pontificia Universidad Católica de Valparaíso, \\ Casilla 4059, Valparaíso, Chile \\ ${ }^{c}$ Universidad Andres Bello, Departamento de Ciencias Físicas, \\ República 220, Santiago, Chile \\ E-mail: mb@ipb.ac.rs, cbranislav@ipb.ac.rs, olivera.miskovic@ucv.cl, \\ rodrigo.olea@unab.cl
}

ABstract: Basic aspects of the AdS/CFT correspondence are studied in the framework of 3-dimensional gravity with torsion. After choosing a consistent holographic ansatz, we formulate an improved approach to the Noether-Ward identities for the boundary theory. The method is applied first to the topological Mielke-Baekler model, and then to the more interesting (parity-preserving) 3-dimensional gravity with propagating torsion. In both cases, we find the finite holographic energy-momentum and spin currents and obtain the associated (anomalous) Noether-Ward identities.

KeYwords: AdS-CFT Correspondence, Spacetime symmetries, Anomalies in Field and String Theories, Classical theories of gravity 


\section{Contents}

1 Introduction 1

2 Holographic ansatz 3

2.1 Restricting the local Poincaré symmetry 3

$\begin{array}{lll}2.2 & \text { Residual gauge symmetries } & 6\end{array}$

$\begin{array}{lll}3 & \text { Noether-Ward identities } & 7\end{array}$

4 Holography in topological 3D gravity with torsion $\quad 10$

$\begin{array}{ll}4.1 \text { Analysis of the field equations } & 11\end{array}$

$\begin{array}{lll}4.2 & \text { Counterterm and boundary currents } & 12\end{array}$

$\begin{array}{lll}4.3 & \text { Boundary symmetries and anomalies } & 13\end{array}$

5 Holography in 3D gravity with propagating torsion $\quad 14$

$\begin{array}{ll}5.1 \text { Lagrangian and the field equations } & 15\end{array}$

$\begin{array}{ll}5.2 \text { Equations of motion } & 16\end{array}$

$\begin{array}{ll}5.3 \text { Boundary currents } & 16\end{array}$

$\begin{array}{lll}5.4 & \text { Renormalized action } & 18\end{array}$

$\begin{array}{lll}5.5 & \text { Boundary symmetries and anomalies } & 19\end{array}$

6 Concluding remarks $\quad 22$

A On the RC geometry in 2D 22

B Residual symmetries to second order 23

C Field strengths and covariant momenta $\quad 24$

$\begin{array}{lll}\text { C.1 Torsion and curvature } & 24\end{array}$

$\begin{array}{ll}\text { C.2 Covariant momenta } & 25\end{array}$

$\begin{array}{ll}\text { D Radial expansion of the field equations } & 26\end{array}$

\section{Introduction}

According to the idea of AdS/CFT correspondence [1], to any asymptotically anti-de Sitter (AdS) gravitational theory on a $(d+1)$-dimensional spacetime $M$, there corresponds a $d$ dimensional conformal field theory (CFT) on the boundary $\partial M$. This duality is of the weak/strong coupling type: the weak coupling regime of the gravitational theory is related to the strong coupling regime of the boundary CFT, and vice versa. 
Following a widely spread belief that general relativity (GR) is the most reliable approach for studying the gravitational phenomena, the analysis of the AdS/CFT correspondence has been carried out mostly in the realm of Riemannian geometry, leading to a number of highly interesting results $[2,3]$. However, one should note that, for nearly five decades, there exists a modern gauge-theoretic conception of gravity, characterized by a Riemann-Cartan geometry of spacetime. In this approach, known as Poincaré gauge theory (PGT) [4-6], both the torsion and the curvature carry the gravitational dynamics. In spite of its well-founded dynamical structure, the use of this framework for studying the AdS/CFT correspondence is still in a rather rudimentary phase. In this regard, we wish to mention the work of Bañados et al. [7], who studied the holographic currents in the 5-dimensional (5D) Chern-Simons gravity with torsion, and the paper of Klemm and Tagliabue [8], investigating the holographic structure of the Mielke-Baekler (MB) model of 3D gravity with torsion [9]. In 4D, Petkou [10] examined holographic aspects of EinsteinCartan theory amended by topological torsional invariants.

In order to properly understand dynamical features of gravity with torsion, one is naturally led to consider technically simplified models with the same conceptual features. An important and useful model of this type is the MB model of topological 3D gravity with torsion [9], introduced in the early 1990s. Further investigations along these lines led to a number of remarkable results; for more details, see [11, 12] and references therein. Of particular interest for our present work is the existence of a holographic structure, as discussed in [8]. However, in the MB model (like in GR with a cosmological constant) there are no propagating degrees of freedom. In order to overcome this unrealistic feature of the gravitational dynamics, a systematic study of 3D gravity with propagating torsion has been recently initiated in [12], see also [13]. The present work is aimed at investigating holographic aspects of 3D gravity with (propagating) torsion, in order to reexamine the compatibility of the concept of torsion with the basic aspects of the AdS/CFT correspondence, and moreover, to understand the dynamical role of the new CFT sources associated with torsion.

The paper is organized as follows. In section 2, we discuss general holographic features of 3D gravity with torsion, with or without the propagating torsion modes. After choosing a suitable ansatz for the gravitational variables, we derive the related consistency conditions, which tell us that the maximal boundary symmetry consists of the local Poincaré transformations and dilatations. In section 3 , we propose an improved treatment of the corresponding Noether-Ward identities for the boundary theory. In section 4, we use this approach to reexamine the holographic structure of the topological 3D gravity with torsion; our results confirm the analysis of Klemm and Tagliabue [8], based on a different technique. Then, in section 5 , we turn to the main subject of the present work - the study of holography in 3D gravity with propagating torsion. We find that the maximal boundary symmetry is reduced by the existence of the conformal anomaly. The improved formalism ensures that these results do not depend on the value of torsion on the boundary.

Our conventions are given by the following rules. In 3D spacetime $M$, the Latin indices $(i, j, k, \ldots)$ refer to the local Lorentz frame, the Greek indices $(\mu, \nu, \rho, \ldots)$ refer to the coordinate frame, the metric components in the local Lorentz frame are $\eta_{i j}=$ 
$(+1,-1,-1)$, totally antisymmetric tensor $\varepsilon^{i j k}$ is normalized by $\varepsilon^{012}=+1$, and symmetric and antisymmetric pieces of a tensor $X_{i j}$ are $X_{(i j)}=\frac{1}{2}\left(X_{i j}+X_{j i}\right)$ and $X_{[i j]}=\frac{1}{2}\left(X_{i j}-X_{j i}\right)$, respectively. Next, the $(1+2)$ decomposition of spacetime is described in terms of the suitable coordinates $x^{\mu}=\left(\rho, x^{\alpha}\right)$, where $\rho$ is a radial coordinate and $x^{\alpha}$ are local coordinates on the boundary $\partial M$; in the local Lorentz frame, this decomposition is expressed by $i=$ $(1, a)$. Then, on 2D boundary $\partial M$ (which is orthogonal to the radial direction), we have $\eta_{a b}=(+1,-1)$ and $\varepsilon^{a b}:=\varepsilon^{a 1 b}$, with $\varepsilon^{02}=+1$. Finally, we use the Stokes theorem in the form $\int \partial_{\lambda} V^{\lambda} d^{3} x=\int V^{\rho} d^{2} x$, where $V^{\lambda}=\left(V^{\rho}, V^{\alpha}\right)$ is a vector density, and $d^{3} x$ and $d^{2} x$ are coherently oriented volume forms on $M$ and $\partial M$, respectively.

\section{Holographic ansatz}

In this section, we introduce a general setting for 3D gravity with torsion and discuss a suitable holographic ansatz for the basic dynamical variables.

Three-dimensional gravity with torsion can be naturally described in the framework of PGT $[11,12]$, where basic gravitational variables are the triad field $\hat{e}^{i}$ and the Lorentz connection $\hat{\omega}^{i j}=-\hat{\omega}^{j i}$ (1-forms), the corresponding field strengths are $\hat{T}^{i}=d \hat{e}^{i}+\hat{\omega}^{i}{ }_{j} \wedge \hat{e}^{j}$ and $\hat{R}^{i j}=d \hat{\omega}^{i j}+\hat{\omega}^{i}{ }_{k} \wedge \hat{\omega}^{k j}$ (2-forms), and the covariant derivative $\hat{\nabla}=d+\frac{1}{2} \hat{\omega}^{i j} \sum_{i j}$ (1-form) acts on local Lorentz spinors/tensors in accordance with their spinorial structure, encoded in the form of the spin matrix $\Sigma_{i j}$. The antisymmetry of $\hat{\omega}^{i j}$ ensures that the underlying geometric structure of spacetime is given by the Riemann-Cartan (RC) geometry, in which $\hat{e}^{i}$ is an orthonormal frame, $\hat{g}=\eta_{i j} \hat{e}^{i} \otimes \hat{e}^{j}$ is the metric of spacetime, $\hat{\omega}^{i j}$ is the metriccompatible connection, $\hat{\nabla} \hat{g}=0$, and $\hat{T}^{i}$ and $\hat{R}^{i j}$ are the torsion and the RC curvature of spacetime, respectively. In our convention, hatted variables are 3D objects. Clearly, general features of PGT make it dynamically quite different from Riemannian theories, such as, for instance, topologically massive gravity $[14,15]$ or the Bergshoeff-Hohm-Townsend gravity [16].

In 3D, to any antisymmetric form $\hat{X}^{i j}$ there corresponds its Lie dual form $\hat{X}_{k}$, defined by $\hat{X}^{i j}=-\varepsilon^{i j k} \hat{X}_{k}$. Replacing $\hat{\omega}^{i j}, \hat{R}^{i j}$ with their Lie duals $\hat{\omega}^{i}, \hat{R}^{i}$, we have:

$$
\hat{T}^{i}=d \hat{e}^{i}+\varepsilon^{i}{ }_{j k} \hat{\omega}^{j} \wedge \hat{e}^{k}, \quad \hat{R}^{i}=d \hat{\omega}^{i}+\frac{1}{2} \varepsilon^{i}{ }_{j k} \hat{\omega}^{j} \wedge \hat{\omega}^{k}
$$

In local coordinates $x^{\mu}$, we can write $\hat{e}^{i}=\hat{e}^{i}{ }_{\mu} d x^{\mu}, \hat{\omega}^{i}=\hat{\omega}^{i}{ }_{\mu} d x^{\mu}$, and the action of local Poincaré transformations on the basic dynamical variables reads:

$$
\begin{aligned}
& \delta_{0} \hat{e}^{i}{ }_{\mu}=-\varepsilon^{i j k} \hat{e}_{j \mu} \hat{\theta}_{k}-\left(\partial_{\mu} \hat{\xi}^{\lambda}\right) \hat{e}_{\lambda}^{i}-\hat{\xi}^{\lambda} \partial_{\lambda} \hat{e}_{\mu}^{i}, \\
& \delta_{0} \hat{\omega}^{i}{ }_{\mu}=-\hat{\nabla}_{\mu} \hat{\theta}^{i}-\left(\partial_{\mu} \hat{\xi}^{\lambda}\right) \hat{\omega}^{i}{ }_{\lambda}-\hat{\xi}^{\lambda} \partial_{\lambda} \hat{\omega}^{i}{ }_{\mu} .
\end{aligned}
$$

Here, $\delta_{0}$ is the form variation of a field, the parameters $\hat{\theta}^{i}$ and $\hat{\xi}^{\mu}$ describe local Lorentz transformations and local translations, respectively, and $\hat{\nabla}_{\mu} \hat{\theta}^{i}=\partial_{\mu} \hat{\theta}^{i}+\varepsilon^{i}{ }_{j k} \hat{\omega}^{j}{ }_{\mu} \hat{\theta}^{k}$.

Specific features of the RC geometry in 2D are described in Appendix A.

\subsection{Restricting the local Poincaré symmetry}

In order to study the holographic structure of 3D gravity with torsion, we assume that spacetime $M$ is a 3D manifold with a boundary $\partial M$ at spatial infinity; more precisely, 
$M$ is asymptotically diffeomorphic to $R \times \partial M$. The gravitational content of $M$ implies that its geometric structure is of the $\mathrm{RC}$ type, whereas its dynamics is determined by choosing an action integral, which produces the field equations. Given the field equations, the asymptotic behavior of $M$ is controlled by the asymptotic conditions. In the asymptotic region, $M$ can be suitably parametrized by the local coordinates $x^{\mu}=\left(\rho, x^{\alpha}\right)$, where $\rho$ is a radial coordinate, such that $\rho=0$ on $\partial M$. The asymptotic conditions are formulated as certain conditions on the gravitational variables $\hat{e}^{i}$ and $\hat{\omega}^{i}$ near the boundary at $\rho=0$.

The (asymptotic) radial foliation of $M$ is an analog of the temporal foliation in the standard canonical formalism, with time line replaced by the radial line; early ideas on dynamical evolutions along spatial directions can be found in [17]. In this framework, Poincaré gauge invariance implies that $\hat{e}_{\rho}^{i}$ and $\hat{\omega}_{\rho}^{i}$ are unphysical variables, so that their values can be fixed by suitable gauge conditions. Although gauge conditions have no influence on the physical content in the bulk, the boundary dynamics is very sensitive to their form. Based on the experience with the Mielke-Baekler (MB) topological model of $3 \mathrm{D}$ gravity with torsion $[8,11]$, we impose the following six gauge conditions:

$$
\begin{aligned}
& \hat{e}_{\rho}^{i}=\left(\hat{e}^{1}{ }_{\rho}, \hat{e}^{a}{ }_{\rho}\right)=\left(\frac{\ell}{\rho}, 0\right), \\
& \hat{\omega}^{i}=\left(\hat{\omega}^{1}{ }_{\rho}, \hat{\omega}^{a}{ }_{\rho}\right)=\left(\frac{p \ell}{2 \rho}, 0\right),
\end{aligned}
$$

which break the Lorentz and the translational gauge invariance; $\ell$ is the AdS radius. As we shall see below, the parameter $p$ controls the strength of both the torsion and the curvature on $M$. Next, we impose an extra condition:

$$
\hat{e}_{\alpha}^{1}=0
$$

which is equivalent to $\hat{e}_{i}{ }^{\rho}=0$ and is known as the "radial gauge" (an analog of the standard "time gauge"). Geometrically, it ensures that the radial direction coincides with the normal to $\partial M$, which greatly simplifies the calculations. In particular, the matrix representation of $\hat{e}^{i}{ }_{\mu}$ becomes block diagonal. Finally, combining (2.4) with a suitable ansatz for $\hat{e}^{a}{ }_{\alpha}$ and $\hat{\omega}^{i}{ }_{\alpha}$, we can write:

$$
\begin{aligned}
& \hat{e}_{\alpha}^{i}=\left(\hat{e}_{\alpha}{ }_{\alpha}, \hat{e}^{a}{ }_{\alpha}\right)=\left(0, \frac{1}{\rho} e^{a}{ }_{\alpha}\right), \\
& \hat{\omega}^{i}{ }_{\alpha}=\left(\hat{\omega}^{1}{ }_{\alpha}, \hat{\omega}^{a}{ }_{\alpha}\right)=\left(\omega_{\alpha}, \frac{1}{\rho} k^{a}{ }_{\alpha}\right),
\end{aligned}
$$

where $e^{a}{ }_{\alpha}(\rho, x), \omega_{\alpha}(\rho, x)$ and $k^{a}{ }_{\alpha}(\rho, x)$ are assumed to be finite and differentiable functions of $\rho$ at $\rho=0$, such that, near the boundary, they have the form

$$
\begin{aligned}
& e^{a}{ }_{\alpha}(\rho, x)=\bar{e}_{\alpha}^{a}(x)+\mathcal{O}(\rho), \\
& \omega_{\alpha}(\rho, x)=\bar{\omega}_{\alpha}(x)+\mathcal{O}(\rho),
\end{aligned}
$$

and similarly for $\kappa^{a}{ }_{\alpha}(\rho, x)$. Here, $\mathcal{O}(\rho)$ tends to zero when $\rho \rightarrow 0$, a bar over $e^{a}{ }_{\alpha}$ denotes the value of $e^{a}{ }_{\alpha}$ at the boundary $\rho=0$, and similarly for $\bar{\omega}_{\alpha}$. Note, in particular, that the 
conditions (2.6) allow the presence of $\rho^{n} \ln \rho$ terms for $n>0$, but not for the leading term $n=0$. The inverse of $\hat{e}_{\mu}^{i}$ has the form

$$
\begin{aligned}
& \hat{e}_{i}{ }^{\rho}=\left(\hat{e}_{1}{ }^{\rho}, \hat{e}_{a}{ }^{\rho}\right)=\left(\frac{\rho}{\ell}, 0\right), \\
& \hat{e}_{i}{ }^{\alpha}=\left(\hat{e}_{1}^{\alpha}, \hat{e}_{a}^{\alpha}\right)=\left(0, \rho e_{a}^{\alpha}\right) .
\end{aligned}
$$

The geometric interpretation of $e^{a}{ }_{\alpha}, \omega_{\alpha}$ and $k^{a}{ }_{\alpha}$ will be given in the next subsection.

Based on these conditions, we will investigate the holographic structure of $3 \mathrm{D}$ gravity with torsion, assuming the absence of matter. In particular, we shall study two complementary dynamical situations, described by

(a) MB model of topological 3D gravity with torsion, and

(b) general (parity-preserving) 3D gravity with propagating torsion.

For later convenience, we note that the metric defined by (2.3) and (2.5),

$$
d s^{2}=\hat{g}_{\mu \nu} d x^{\mu} d x^{\nu}=-\frac{\ell^{2} d \rho^{2}}{\rho^{2}}+\frac{1}{\rho^{2}} g_{\alpha \beta} d x^{\alpha} d x^{\beta},
$$

where $g_{\alpha \beta}:=e^{a}{ }_{\alpha} e^{b}{ }_{\beta} \eta_{a b}$ is regular at $\rho=0$ and takes the usual Fefferman-Graham form [18]. For $\rho=0$, the full metric has a pole of order two, which is typical for asymptotically AdS spaces, and directly related to the pole of order one in the triad field (2.5a).

In the rest of the paper, we use the units in which the AdS radius is $\ell=1$.

Comment on (2.6). Any assumption on the asymptotic form of dynamical variables restricts the set of possible solutions of the field equations. In general, depending on the model-dependent dynamical features, expansions of the fields in (2.6) could contain logarithmic terms or power series of different order. However, having in mind that the holographic structure of the general 3D gravity model (b) has not been studied before, our intention is not to make the most general holographic analysis, which would be technically extremely complex, but to identify its basic holographic features. Furthermore, since both models (a) and (b) possess asymptotically AdS black hole solution [12], it is quite natural to expect that those features can be successfully revealed by focusing on the AdS asymptotic sector of the Brown-Henneaux type [11, 20].

To be more specific, let us mention that certain holographic aspects of the MB model in the Chern-Simons formulation have been studied earlier by Klemm and Tagliabue [8]. Their results strongly suggest that, in the MB model, our assumption (2.6) should be restricted to the following form:

$$
\begin{aligned}
& \hat{e}^{a}{ }_{\alpha}(\rho, x)=\bar{e}^{a}{ }_{\alpha}+\rho^{2} s^{a}{ }_{\alpha}+\mathcal{O}_{4}, \\
& \hat{\omega}_{\alpha}(\rho, x)=\bar{\omega}_{\alpha}+\mathcal{O}_{2},
\end{aligned}
$$

where $\mathcal{O}_{n}$ is a term that tends to zero as $\rho^{n}$ or faster, when $\rho \rightarrow 0$. Moreover, we expect the same sector to be of prime interest for the holographic structure of the general 3D gravity model (b). As we shall see, the results obtained in sections 4 and 5 justify our expectations. In this section, however, we continue using only (2.6). 


\subsection{Residual gauge symmetries}

A field theory is defined by both the field equations and the asymptotic (boundary) conditions. The concept of asymptotic symmetry is of fundamental importance for understanding basic aspects of the boundary dynamics. Since the conditions (2.3), (2.5) and (2.6) control the form of dynamical variables in the asymptotic region near $\rho=0$, they have a decisive influence on the asymptotic symmetry. The asymptotic symmetry is defined by a subset of gauge transformations that leaves the asymptotic conditions invariant. Thus, the parameters of the asymptotic (or residual) gauge transformations are defined by the consistency requirements

$$
\begin{aligned}
& -\varepsilon^{i j k} \hat{e}_{j \mu} \hat{\theta}_{k}-\left(\partial_{\mu} \hat{\xi}^{\lambda}\right) \hat{e}_{\lambda}^{i}-\hat{\xi}^{\lambda} \partial_{\lambda} \hat{e}^{i}{ }_{\mu}=0, \\
& -\nabla_{\mu} \hat{\theta}^{i}-\left(\partial_{\mu} \hat{\xi}^{\lambda}\right) \hat{\omega}^{i}{ }_{\lambda}-\hat{\xi}^{\lambda} \partial_{\lambda} \hat{\omega}^{i}{ }_{\mu}=0,
\end{aligned}
$$

where $\hat{e}_{\mu}^{i}$ and $\hat{\omega}^{i}{ }_{\mu}$ are taken to satisfy (2.3) and (2.5).

Starting with these conditions, we first find the restrictions stemming from the invariance of $\hat{e}^{1}{ }_{\rho}, \hat{e}^{a}{ }_{\rho}, \hat{e}^{1}{ }_{\alpha}$, and $\hat{\omega}^{1}{ }_{\rho}$, respectively:

$$
\begin{aligned}
& \hat{\xi}^{\rho}=\rho f(x), \\
& \partial_{\rho} \hat{\xi}^{\alpha}=\rho g^{\alpha \beta} \partial_{\beta} f, \\
& \hat{\theta}^{a}=\rho \varepsilon^{a b} e_{b}{ }^{\alpha} \partial_{\alpha} f, \\
& \partial_{\rho} \hat{\theta}^{1}=-\rho \omega^{\alpha} \partial_{\alpha} f .
\end{aligned}
$$

There relations give rise to the following radial radial expansion of the local parameters:

$$
\begin{aligned}
& \hat{\xi}^{\rho}=\rho f(x), \\
& \hat{\xi}^{\alpha}=\xi^{\alpha}(x)+\frac{1}{2} \rho^{2} \bar{g}^{\alpha \beta} \partial_{\beta} f+\rho^{2} \mathcal{O}(\rho), \\
& \hat{\theta}^{a}=\rho \varepsilon^{a b} \bar{e}_{b}^{\alpha} \partial_{\alpha} f+\rho \mathcal{O}(\rho), \\
& \hat{\theta}^{1}=\theta(x)-\frac{\rho^{2}}{2} \bar{\omega}^{\alpha} \partial_{\alpha} f+\rho \mathcal{O}\left(\rho^{2}\right) .
\end{aligned}
$$

Thus, the residual symmetry is expressed in terms of the four boundary parameters: $\xi^{\alpha}(x), \theta(x)$ and $f(x)$.

In the next step, we find the restrictions produced by the invariance of $\hat{\omega}^{a}{ }_{\rho}$ and $\hat{\omega}^{a}{ }_{\alpha}$, respectively:

$$
\begin{aligned}
& {\left[\left(\varepsilon^{a b}-\frac{p}{2} \eta^{a b}+k^{a b}\right) e_{b}{ }^{\beta}+\rho \varepsilon^{a b}\left(\partial_{\rho} e_{b}{ }^{\beta}\right)\right] \partial_{\beta} f=0,} \\
& \delta_{0} k^{a}{ }_{\alpha}=\left[-\varepsilon^{a b} k_{b \alpha} \theta-\left(\partial_{\alpha} \xi^{\beta}\right) k^{a}{ }_{\beta}-\xi^{\beta} \partial_{\beta} k^{a}{ }_{\alpha}\right]+f k^{a}{ }_{\alpha}+\mathcal{O}(\rho) .
\end{aligned}
$$

Assuming that $f(x)$ is an arbitrary function on $\partial M$, we have $\partial_{\beta} f \neq 0$, and the first relation defines $k^{a}{ }_{\alpha}$ in terms of the $e^{a}{ }_{\alpha}$ :

$$
k^{a b}=\frac{p}{2} \eta^{a b}-\varepsilon^{a b}-\rho \varepsilon^{a c} e_{c}{ }^{\beta} \partial_{\rho} e^{b}{ }_{\beta},
$$

where $k^{a b}=k^{a}{ }_{\alpha} e_{b}{ }^{\alpha}$. The second relation in (2.10) defines the transformation law for $k^{a}{ }_{\alpha}$; it shows that, at the boundary, $k^{a}{ }_{\alpha}$ is a tensorial object with respect to local Poincaré 
transformations combined with dilatations. As shown in Appendix A, $K_{a b}=\varepsilon_{c b} k_{a}^{c}$ is the extrinsic curvature of $\partial M$.

Finally, we wish to examine the implications of the invariance conditions for $\hat{e}^{a}{ }_{\alpha}$ and $\hat{\omega}^{1}{ }_{\alpha}$. Using (2.5), these condition yield, in the lowest order of the radial expansion, the following transformation rules for the boundary fields:

$$
\begin{aligned}
& \delta_{0} \bar{e}^{a}{ }_{\alpha}=\delta_{P} \bar{e}^{a}{ }_{\alpha}+f \bar{e}^{a}{ }_{\alpha}, \\
& \delta_{0} \bar{\omega}_{\alpha}=\delta_{P} \bar{\omega}_{\alpha}+\varepsilon_{a b} \bar{e}^{a}{ }_{\alpha} \bar{e}^{b \beta} \partial_{\beta} f,
\end{aligned}
$$

where $\delta_{P} \bar{e}^{a}{ }_{\alpha}$ and $\delta_{P} \bar{\omega}_{\alpha}$ are the local Poincaré transformations in 2D:

$$
\begin{aligned}
& \delta_{P} \bar{e}^{a}{ }_{\alpha}=-\varepsilon^{a}{ }_{c} \theta \bar{e}^{c}{ }_{\alpha}-\left(\partial_{\alpha} \xi^{\beta}\right) \bar{e}^{a}{ }_{\beta}-\xi \cdot \partial \bar{e}^{a}{ }_{\alpha}, \\
& \delta_{P} \bar{\omega}_{\alpha}=-\partial_{\alpha} \theta-\left(\partial_{\alpha} \xi^{\beta}\right) \bar{\omega}_{\beta}-\xi \cdot \partial \bar{\omega}_{\alpha},
\end{aligned}
$$

and $f$ defines local dilatations. Thus, we conclude the following:

- The residual symmetry transformations (2.12) belong to the Weyl group of local Poincaré transformations plus dilatations, whereas $\bar{e}^{a}{ }_{\alpha}$ and $\bar{\omega}_{\alpha}$ are recoginzed as the vielbein and the spin connection of the boundary RC geometry.

The transformation rule for $\bar{e}^{a}{ }_{\alpha}$ can be used to calculate how the residual symmetries act on the boundary metric $\bar{g}_{\alpha \beta}=\eta_{a b} \bar{e}^{a}{ }_{\alpha} \bar{e}^{b}{ }_{\beta}$. Restricting our attention to dilatations $(f \neq 0)$, we obtain $\delta_{f} \bar{g}_{\alpha \beta}=2 f \bar{g}_{\alpha \beta}$. For more details, see Appendix B.

The results obtained in this subsection are based only on the adopted holographic conditions (2.3), (2.5) and (2.6). We consider them as being kinematical, in the sense that they are not influenced by the dynamical arguments encoded in (2.8). Another useful set of kinematical relations is found by calculating the expressions for the torsion and the curvature tensors, based on (2.3), (2.5) and (2.6). As shown in Appendix C.1, the result is of the form

$$
\hat{T}_{i j k}=p \varepsilon_{i j k}+\mathcal{O}(\rho), \quad \hat{R}_{i j k}=q \varepsilon_{i j k}+\mathcal{O}(\rho),
$$

where

$$
q:=\frac{p^{2}}{4}-1
$$

Thus, to lowest order in $\rho$, the parameter $p$ defines both the torsion and the curvature of spacetime.

In sections 4 and 5, we shall combine these results with (2.8) to study the specific dynamical models.

\section{Noether-Ward identities}

It is clear from the previous discussion that the residual gauge symmetries (2.9) are also kinematical. They are maximal gauge symmetries that we can expect to find on the boundary. Indeed, after choosing an action integral, the corresponding field equations may impose additional restrictions on these symmetries. In this section, we shall study the 
gravitational Noether identities (also called generalized conservation laws) induced by the maximal gauge symmetries (2.9), and interpret them as the corresponding Ward identities of the boundary CFT.

To make these ideas more precise, consider a 3D gravitational system without matter in an asymptotically AdS spacetime, with solutions characterized by independent boundary values of $e^{a}{ }_{\alpha}$ and $\omega_{\alpha}$. The quasilocal energy-momentum and spin currents of the system are calculated by varying the action with respect to the boundary values of $e^{a}{ }_{\alpha}$ and $\omega_{\alpha}$. The variation produces a bulk term, which is proportional to the field equations, and a boundary term. The on-shell value of the gravitational action, suitably renormalized, is given as a finite 2D functional $I_{\text {ren }}[e, \omega]$ on $\partial M$. Next, consider a set of quantum fields $\phi$ on $\partial M$, coupled to the external gravitational fields (sources) $e^{a}{ }_{\alpha}$ and $\omega_{\alpha}$, and described by an action integral $I[\phi ; e, \omega]$. The corresponding effective action $W[e, \omega]$ is defined by the functional average over $\phi$ :

$$
e^{i W[e, \omega]}=\int_{\partial M} D \phi e^{i I[\phi ; e, \omega]} .
$$

In the semiclassical approximation, the AdS/CFT correspondence can be expressed by identifying the effective action with $I_{\mathrm{ren}}[e, \omega]$ :

$$
W[e, \omega]=I_{\mathrm{ren}}[e, \omega] .
$$

Using this identification, we can calculate the gravitational Noether identities for $I_{\text {ren }}[e, \omega]$ and identify them as the Ward identities for the 1-point functions derived from $W[e, \omega]$, provided the functional measure is invariant under the residual gauge symmetries.

We consider gravity theories whose Lagrangians are at most quadratic in the first derivatives of the spin connection and the vielbein. The corresponding field equations are obtained integrating by parts, such that the surface term,

$$
\delta I_{\mathrm{on}-\mathrm{shell}}=\int d^{2} x\left(P_{i}^{\nu} \delta \hat{e}^{i}{ }_{\nu}+Q_{i}^{\nu} \delta \hat{\omega}_{\nu}^{i}\right)
$$

does not contain derivatives of the variations of the fields.

The gauge choice (2.3)-(2.5), when used in the above formula, produces a surface term expressed in terms of the boundary quantities

$$
\delta I_{\mathrm{on}-\text { shell }}=\int d^{2} x\left(p_{i}^{\alpha} \delta e_{\alpha}^{a}+q^{\alpha} \delta \omega_{\alpha}+\tilde{q}_{a}^{\alpha} \delta k_{\alpha}^{a}\right) .
$$

It is clear that the PGT formulation of gravity also allows to impose boundary conditions different than keeping the vielbein and spin connection fixed at the conformal boundary. However, a theory with boundary conditions other than a Dirichlet one does not lead itself to a holographic description in the usual AdS/CFT framework.

In fact, in the metric formalism, the last term in (3.3) is related to the variation of the extrinsic curvature that is usually traded off for the variation of metric by a GibbonsHawking-type term. When a Gibbons-Hawking-type term cannot be constructed for a given theory, the only way out is to consider that the extrinsic curvature and the metric are related asymptotically. 
The fact that the leading-order in the expansion of the extrinsic curvature is the same as the leading-order of the boundary metric for Riemannian AdS spacetimes suggests that there is an asymptotic relation between the extrinsic curvature and the vielbein in theories with torsion; such a relation in PGT is given by (2.11). Note that, as showed in Appendix A, only the symmetric part of the extrinsic curvature is Riemannian, and the antisymmetric one explicitly depends on torsion. Once appropriate counterterms are added, the variation of the renormalized PGT action can be written as

$$
\delta I_{\mathrm{ren}}=-\int_{\partial M} d^{2} x\left(\tau^{\alpha}{ }_{a} \delta e^{a}{ }_{\alpha}+\sigma^{\alpha} \delta \omega_{\alpha}\right),
$$

whereby the standard duality between gravity and a boundary CFT is recovered.

The form of the expected Noether identities is based on the residual symmetry transformations (2.12) and (2.13). Quite generally, the invariance of the renormalized action under these transformations can be written in the form

$$
\delta I_{\text {ren }}=-\int_{\partial M} d^{2} x\left(\tau^{\alpha}{ }_{a} \delta_{0} e^{a}{ }_{\alpha}+\sigma^{\alpha} \delta_{0} \omega_{\alpha}\right)=0
$$

where

$$
\tau_{a}^{\alpha}:=-\frac{\delta I_{\mathrm{ren}}}{\delta e^{a}{ }_{\alpha}}, \quad \sigma^{\alpha}:=-\frac{\delta I_{\mathrm{ren}}}{\delta \omega_{\alpha}},
$$

are the energy-momentum and spin currents (tensor densities) of our dynamical system.

Restricting our attention first to the local translations (with parameters $\xi^{\alpha}$ ) and then to the local Lorentz transformations (with parameter $\theta$ ), we arrive at the corresponding Noether identities:

$$
\begin{aligned}
& e^{a}{ }_{\beta} \nabla_{\alpha} \tau^{\alpha}{ }_{a}=\tau^{\alpha}{ }_{a} T^{a}{ }_{\beta \alpha}+\sigma^{\alpha} F_{\beta \alpha}-\omega_{\beta}\left(\nabla_{\alpha} \sigma^{\alpha}+\varepsilon^{a b} \tau_{a b}\right), \\
& \nabla_{\beta} \sigma^{\beta}=-\varepsilon^{a b} \tau_{a b},
\end{aligned}
$$

which are also known as the generalized conservation laws of $\tau^{\alpha}{ }_{a}$ and $\sigma^{\beta}$. Note that if the second Noether identity (3.6b) is fulfilled, the last term in (3.6a) can be omitted. Similarly, the invariance of $I_{\mathrm{ren}}\left[e^{a}{ }_{\alpha}, \omega_{\alpha}\right]$ under dilatations leads to

$$
\tau-\nabla_{\beta}\left(\varepsilon_{a b} \sigma^{a} e^{b \beta}\right)=0,
$$

where $\tau:=\tau_{a}^{a}$ is the trace of the energy momentum tensor.

Although the gravitational dynamics in the bulk is described by a RC geometry, with $\hat{\omega}^{i}{ }_{\mu}$ and $\hat{e}^{i}{ }_{\mu}$ as independent fields, it may happen that some solutions on the boundary are Riemannian, that is, characterized by a vanishing torsion, $T_{a b c}=0$. For such solutions, the boundary connection $\omega_{\alpha}$ is no longer independent of the vielbein $e^{a}{ }_{\alpha}$. Nevertheless, as we are going to show, the Noether-Ward identities are still of the form (3.6), but now, $\omega_{\alpha}$ takes on the Riemannian value $\tilde{\omega}_{\alpha}$. In a way, this might have been expected, since the transformation properties of $\tilde{\omega}_{\alpha}$ are the same as those of $\omega_{\alpha}$, and these properties play a crucial role in defining the boundary symmetry.

When the boundary torsion vanishes, the connection takes the Riemannian form (A.3). However, we find it more convenient to use an equivalent but more compact expression:

$$
\tilde{\omega}_{\alpha}=-\varepsilon_{a b} \varepsilon^{\gamma \delta} \varepsilon_{\alpha \beta} e^{a \beta} \partial_{\gamma} e^{b}{ }_{\delta} .
$$


Now, starting from the Riemannian renormalized action $\tilde{I}_{\text {ren }}=I_{\text {ren }}\left[e^{a}{ }_{\alpha}, \tilde{\omega}_{\alpha}\right]$, we find that the related spin current $\Sigma^{\alpha}:=-\delta \tilde{I}_{\text {ren }} / \delta \omega_{\alpha}$ vanishes, whereas the energy-momentum current $\Theta^{\alpha}{ }_{a}:=-\delta \tilde{I}_{\text {ren }} / \delta e^{a}{ }_{\alpha}$ has an additional contribution stemming from the last term in (3.5a):

$$
\Theta^{\alpha}{ }_{a}=\tilde{\tau}_{a}^{\alpha}-\tilde{\nabla}_{\beta}\left(\varepsilon^{\alpha \beta} e^{-1} \tilde{\sigma}_{a}\right) .
$$

Here, $\tilde{X}$ denotes the Riemannian limit of a RC object $X$; in particular, $\tilde{\nabla}_{\alpha} f_{a}=\partial_{\alpha} f_{a}-$ $\varepsilon_{a c} \tilde{\omega}_{\alpha} f^{c}$. Then, the Noether identities for the action $\tilde{I}_{\text {ren }}$ are found to be:

$$
\begin{aligned}
& e^{a}{ }_{\beta} \tilde{\nabla}_{\alpha} \Theta^{\alpha}{ }_{a}+\tilde{\omega}_{\beta} \varepsilon^{a b} \Theta_{a b}=0, \\
& \varepsilon^{a b} \Theta_{a b}=0, \\
& \Theta=0 .
\end{aligned}
$$

Since $\Theta^{\alpha}{ }_{a}$ is a tensor density, the first relation, which is a condition for diffeomorphism invariance, is seen to coincide with the condition (4.10) in Klemm et al. [8]. When the Lorentz invariance is satisfied, (3.9a) reduces to the usual form $D_{\alpha}\left(e^{-1} \Theta^{\alpha}{ }_{\beta}\right)=0$, where $D_{\alpha}$ is the Riemannian covariant derivative. The remaining two relations are the standard Riemannian conditions for the Lorentz and Weyl invariance, respectively. Using $T_{a b c}=0$, as well as the identity $\varepsilon^{\alpha \beta} \tilde{\nabla}_{\alpha} \tilde{\nabla}_{\beta} f_{a}=-\frac{1}{2} \varepsilon^{\alpha \beta} \tilde{F}_{\alpha \beta} \varepsilon_{a b} f^{b}$, one can transform (3.9) into

$$
\begin{aligned}
& e^{a}{ }_{\beta} \tilde{\nabla}_{\alpha} \tilde{\tau}^{\alpha}{ }_{a}-\tilde{\sigma}^{\alpha} F_{\beta \alpha}+\tilde{\omega}_{\beta}\left(\tilde{\nabla}_{\beta} \tilde{\sigma}^{\beta}+\varepsilon^{a b} \tilde{\tau}_{a b}\right)=0, \\
& \varepsilon^{a b} \tilde{\tau}_{a b}+\tilde{\nabla}_{\beta} \tilde{\sigma}^{\beta}=0, \\
& \tilde{\tau}-\tilde{\nabla}_{\beta}\left(\varepsilon_{a b} \tilde{\sigma}^{a} e^{b \beta}\right)=0 .
\end{aligned}
$$

Hence, the Riemannian identities (3.9) coincide with those obtained from (3.6) in the limit $T_{a b c} \rightarrow 0$, as expected. This proves the following theorem:

- In the context of PGT, the form (3.6) of Noether identities can be used for both Riemann-Cartan and Riemannian boundary geometries.

According to the AdS/CFT correspondence, relations (3.6) are interpreted as the maximal set of Ward identities that can be found in the boundary CFT. If the field equations happen to be incompatible with the above symmetries, some of the Ward identities may be violated, leading to the appearance of quantum anomalies.

\section{Holography in topological 3D gravity with torsion}

In this section, we analyze the validity of the Noether-Ward identities (3.6), in the MB model of topological 3D gravity with torsion [9, 11], described by the action

$$
I_{\mathrm{MB}}=\int\left(2 a \hat{e}^{i} \hat{R}_{i}-\frac{1}{3} \Lambda_{0} \varepsilon_{i j k} \hat{e}^{i} \hat{e}^{j} \hat{e}^{k}+\alpha_{3} L_{\mathrm{CS}}(\hat{\omega})+\alpha_{4} \hat{e}^{i} \hat{T}_{i}\right)
$$

where $L_{\mathrm{CS}}(\hat{\omega})=\hat{\omega}_{i} d \hat{\omega}^{i}+\frac{1}{3} \varepsilon_{i j k} \hat{\omega}^{i} \hat{\omega}^{j} \hat{\omega}^{k}$ is the Chern-Simons Lagrangian for the Lorentz connection, $a=1 / 16 \pi G$ is the gravitational constant, $\Lambda_{0}$ is a (bare) cosmological constant, 
$\alpha_{3}, \alpha_{4}$ are dimensionful coupling constants, the wedge product signs $\wedge$ are omitted for simplicity, and the matter contribution is absent.

The vacuum field equations read

$$
\hat{T}_{i j k}=p \varepsilon_{i j k}, \quad \hat{R}_{i j k}=q \varepsilon_{i j k},
$$

where the parameters $p$ and $q$ are defined in terms of the coupling constants $a, \Lambda, \alpha_{3}, \alpha_{4}$. The spacetime described by these equations is maximally symmetric, at least locally. Moreover, in the AdS sector, the effective cosmological constant is negative,

$$
\Lambda_{\mathrm{eff}}:=q-\frac{p^{2}}{4}=-1
$$

By comparing these equations with (2.14), it follows that the parameter $p$ from our ansatz should be identified with the parameter $p$ in the MB model.

Our analysis is based on using the AdS asymptotic conditions (2.8). For an interesting asymptotic correspondence between the MB model and topologically massive gravity, see $[21]$.

\subsection{Analysis of the field equations}

The subset of the field equations (4.2a) that describes the radial evolution of the system is given by $(i j k)=(11 c),(a 1 c)$. The first pair of equations takes the form

$$
\hat{T}_{11 c}=0, \quad \hat{R}_{11 c}=0 .
$$

Using the expressions for $\hat{T}_{i j k}$ and $\hat{R}_{i j k}$ calculated in Appendix C, one finds that the first equation is identically satisfied, whereas the second one implies that $\omega_{\alpha}$ is the Lorentz connection at the boundary,

$$
\omega_{\alpha}=\omega_{\alpha}(x)
$$

The second pair of equations reads:

$$
\hat{T}_{a 1 c}=-p \varepsilon_{a c}, \quad \hat{R}_{a 1 c}=-q \varepsilon_{a c} .
$$

After introducing the radial expansion (2.6), the first equation in (4.4a) yields that $s_{a b}$ is symmetric,

$$
\varepsilon^{a b} s_{a b}=0 .
$$

This result simplifies the second equation in (4.4a); relying on (C.5) $)_{3}$, the piece of the zeroth order in $\rho$ implies that the effective cosmological constant $\Lambda_{\text {eff }}$ is negative, see (4.2b), whereas the piece of order $\rho^{2}$ leads to a finite radial expansion of $e_{c \beta}$ :

$$
e_{c \beta}=\bar{e}_{c \beta}+\rho^{2} \bar{s}_{c \beta} \text {. }
$$

Such an expansion that terminates at $\rho^{2}$ is a generalization of the result known for GR in $3 \mathrm{D}$; in higher dimensions, the result holds when the Weyl tensor vanishes [22]. As a simple consequence, the radial expansion of $k^{a b}$ is also finite:

$$
k^{a b}=\frac{p}{2} \eta^{a b}-\varepsilon^{a b}+2 \rho^{2} \varepsilon^{a c} s_{c}^{b} .
$$


Using the above results, the nontrivial content of the remaining $(1 b c)$ and $(a b c)$ field equations is expressed in terms of the following radial contraints:

$$
\begin{aligned}
& T_{a b c}=0, \\
& R-4 s^{c}{ }_{c}=\mathcal{O}_{2}, \\
& \nabla_{\alpha} s_{b \beta}-\nabla_{\beta} s_{b \alpha}=0 .
\end{aligned}
$$

In particular, we see that the boundary torsion vanishes.

\subsection{Counterterm and boundary currents}

Now, we introduce the boundary currents and verify their Noether-Ward identities.

The variation of the MB action, calculated on shell, reduces to a surface integral:

$$
\delta I_{\mathrm{MB}}=\int_{\partial M} d^{2} x \varepsilon^{\alpha \beta}\left(2 a \hat{e}_{\alpha}^{i} \delta \hat{\omega}_{i \beta}+\alpha_{3} \hat{\omega}^{i}{ }_{\alpha} \delta \hat{\omega}_{i \beta}+\alpha_{4} \hat{e}_{\alpha}^{i} \delta \hat{e}_{i \beta}\right) .
$$

Each of these three terms can be written in more details as:

$$
\begin{aligned}
2 a \varepsilon^{\alpha \beta} \hat{e}^{i}{ }_{\alpha} \delta \hat{\omega}_{i \beta}= & \frac{2 a}{\rho^{2}} \varepsilon^{\alpha \beta}\left[\frac{p}{2} e^{b}{ }_{\alpha} \delta e_{b \beta}-\varepsilon_{a b} e^{a}{ }_{\alpha} \delta e^{b}{ }_{\beta}-2 \rho^{2} \varepsilon_{a b} s^{a}{ }_{\alpha} \delta e^{b}{ }_{\beta}\right]+\delta \Delta_{1}, \\
\alpha_{3} \varepsilon^{\alpha \beta} \hat{\omega}^{i}{ }_{\alpha} \delta \hat{\omega}_{i \beta}= & \frac{\alpha_{3}}{\rho^{2}} \varepsilon^{\alpha \beta}\left[q e^{b}{ }_{\alpha} \delta e_{b \beta}-2 p \rho^{2} \varepsilon^{a b} s_{a \alpha} \delta e_{b \beta}+4 \rho^{2} s^{b}{ }_{\alpha} \delta e_{b \beta}\right] \\
& -\alpha_{3} \varepsilon^{\alpha \beta} \omega_{\alpha} \delta \omega_{\beta}, \\
\alpha_{4} \varepsilon^{\alpha \beta} \hat{e}^{i}{ }_{\alpha} \delta \hat{e}_{i \beta}= & \frac{\alpha_{4}}{\rho^{2}} \varepsilon^{\alpha \beta} e^{b}{ }_{\alpha} \delta e_{b \beta},
\end{aligned}
$$

where $\delta \Delta_{1}$ is a total variation with

$$
\Delta_{1}:=4 a \varepsilon^{\alpha \beta} \varepsilon_{a b} s^{a}{ }_{\alpha} e^{b}{ }_{\beta}=-4 a \bar{e} s_{c}^{c},
$$

and $e:=\operatorname{det}\left(e^{a}{ }_{\alpha}\right)$. Then, the identity $a p+\alpha_{3} q+\alpha_{4}=0$, see Ref. [11], implies that the sum of the first three terms in the above expressions vanishes, whereupon the only divergent term in $\delta I_{\mathrm{MB}}$ is also a total variation, $\delta \Delta_{2}$, with

$$
\Delta_{2}:=-\frac{a}{\rho^{2}} \varepsilon^{\alpha \beta} \varepsilon_{a b} e^{a}{ }_{\alpha} e^{b}{ }_{\beta}=\frac{2 a}{\rho^{2}} \bar{e}\left(1+\rho^{2} s_{c}^{c}\right) .
$$

Since the boundary integral of $\Delta_{1}+\Delta_{2}$ appears in $\delta I_{\mathrm{MB}}$ as a total variation, it can be subtracted from $I_{\mathrm{MB}}$ to obtain an improved variational principle. The integral

$$
I_{\mathrm{ct}}:=\int_{\partial M} d^{2} x\left(\Delta_{1}+\Delta_{2}\right)=2 a \int_{\partial M} d^{2} x \bar{e}\left(\frac{1}{\rho^{2}}-s^{c}{ }_{c}\right)
$$

is usually called the counterterm. Before discussing its role in the new variational principle, let us rewrite $I_{\mathrm{ct}}$ in an equivalent form as

$$
I_{\mathrm{ct}}:=a \int_{\partial M} d^{2} x \tilde{e} K,
$$

where $K$ is the trace of the extrinsic curvature (A.5), and $\tilde{e}^{a}{ }_{\alpha}=\bar{e}^{a}{ }_{\alpha} / \rho$ is the induced vielbein at the boundary. The expression for $I_{\mathrm{ct}}$ is just one-half of the Gibbons-Hawking 
term $\left(I_{\mathrm{GH}}\right)$, the result that naturally appears in the Chern-Simons formulation of GR in 3D, as discussed by Bañados and Méndez [23], and by Mišković and Olea [24] (for an interesting approach to counterterms in higher dimensional gravity, see [25]). On the other hand, using the field equation (4.5b), we can express the finite piece of the counterterm, $s^{c}{ }_{c}$, in terms of the scalar curvature $R$, but since $R$ is a topological invariant, its contribution to $I_{\text {ct }}$ can be disregarded. Thus, effectively, the counterterm can be written as a covariant object, determined by a local function of $\tilde{e}^{a}{ }_{\alpha}$ :

$$
I_{\mathrm{ct}}=2 a \int d^{2} x \tilde{e}=I_{\mathrm{GH}}-2 a \int d^{2} x \tilde{e}
$$

where the last term is the usual local counterterm of Balasubramanian and Kraus [26], obtained in the context of 3D GR. It is interesting to note that the nonlinear ChernSimons term in the MB action does not contribute to the counterterm, in agreement with the analysis of [7].

Since the total variation $\delta I_{\mathrm{ct}}$ is a divergent piece of $\delta I_{\mathrm{MB}}$, we are quite naturally led to introduce the renormalized (or, more precisely, the improved) MB action:

$$
I_{\mathrm{MB}}^{\mathrm{ren}}:=I_{\mathrm{MB}}-I_{\mathrm{ct}},
$$

such that it has well-defined functional derivatives and produces finite boundary currents, on-shell.

Note that, although the counterterm (4.7a) ensures that the variation of $I_{\mathrm{MB}}^{\mathrm{ren}}$ is finite and differentiable, one can verify that the value of the renormalized action $I_{\mathrm{MB}}^{\mathrm{ren}}$ is logarithmically divergent. Similarly as in GR, the logarithmic term is proportional to the Euler topological invariant $e R$, which is why it does not influence the variation of $I_{\mathrm{MB}}^{\mathrm{ren}}$. The logarithmic terms, even though topological in three dimensions, are important to be included, because the renormalized gravitational action is identified with the free energy in the dual boundary field theory.

Finally, by noting that

$$
\delta I_{\mathrm{MB}}^{\mathrm{ren}}=\int_{\partial M} d^{2} x \varepsilon^{\alpha \beta}\left[-4\left(a+\frac{\alpha_{3} p}{2}\right) \varepsilon_{a b} s_{\alpha}^{a} \delta e^{b}{ }_{\beta}+4 \alpha_{3} s_{b \alpha} \delta e_{\beta}^{b}-\alpha_{3} \omega_{\alpha} \delta \omega_{\beta}\right],
$$

we can use (3.5b) to obtain the energy-momentum and spin currents on the boundary:

$$
\begin{aligned}
& \tau_{b}^{\beta}=4\left(a+\frac{\alpha_{3} p}{2}\right) \varepsilon^{\alpha \beta} \varepsilon_{a b} s_{\alpha}^{a}-4 \alpha_{3} \varepsilon^{\alpha \beta} s_{b \alpha}, \\
& \sigma^{\beta}=-\alpha_{3} \varepsilon^{\beta \alpha} \omega_{\alpha} .
\end{aligned}
$$

\subsection{Boundary symmetries and anomalies}

Now, we wish to check the expected Noether-Ward identities (3.6).

Using the radial constraints (4.5), we find the following on-shell relations:

$$
\nabla_{\beta} \tau_{b}^{\beta}=0, \quad \nabla_{\beta} \sigma^{\beta}=-\frac{1}{2} \varepsilon^{b c} \tau_{b c} .
$$


Comparing with (3.6b), we see that the Lorentz invariance of the effective $2 \mathrm{D}$ theory is violated, and the Lorentz anomaly reads:

$$
A_{\mathrm{L}}:=\nabla_{\beta} \sigma^{\beta}+\varepsilon^{b c} \tau_{b c}=\frac{1}{2} \varepsilon^{b c} \tau_{b c}=-\frac{1}{2} \alpha_{3} \bar{e} R .
$$

The coefficient $\alpha_{3}$, multiplying the topological (Euler) density $\bar{e} R$, is proportional to the difference of the classical central charges $c^{\mp}$ of the Mielke-Baekler model [11]:

$$
c^{\mp}=24 \pi\left[a \ell+\alpha_{3}\left(\frac{p \ell}{2} \mp 1\right)\right] \text {. }
$$

Next, $(4.11)_{1}$ implies that the translation invariance condition $(3.6 \mathrm{~b})$ is reduced to the form $0=\sigma^{\beta} F_{\alpha \beta}+\omega_{\alpha} \nabla_{\beta} \sigma^{\beta}$. Using the relations

$$
\nabla_{\beta} \sigma^{\beta}=\frac{1}{2} \alpha_{3} e R, \quad \sigma^{\beta} F_{\alpha \beta}=-\frac{1}{2} \alpha_{3} \omega_{\alpha} e R,
$$

we conclude that local translations are a correct boundary symmetry. Hence, there is no translational anomaly:

$$
A_{\mathrm{T}}:=e^{a}{ }_{\beta} \nabla_{\alpha} \tau^{\alpha}{ }_{a}-\tau_{a}^{\alpha} T_{\beta \alpha}^{a}-\sigma^{\beta} F_{\alpha \beta}+\omega_{\alpha}\left(\nabla_{\beta} \sigma^{\beta}+\varepsilon^{a b} \tau_{a b}\right)=0 .
$$

Finally, in order to verify the Noether identity for dilatations (3.6c), we use (4.4b) and (4.5b) to obtain

$$
\begin{aligned}
& \tau_{c}^{c}=-4 \bar{e}\left(a+\frac{\alpha_{3} p}{2}\right) s_{c}^{c}=-\bar{e}\left(a+\frac{\alpha_{3} p}{2}\right) R, \\
& \nabla_{\beta}\left(\varepsilon_{a b} \sigma^{a} e^{b \beta}\right)=-\alpha_{3} \partial_{\beta}\left(\bar{e} \omega^{\beta}\right) .
\end{aligned}
$$

Thus, the dilatational Noether identity is violated, and the violation is measured by a quantity which is usually called the conformal (or Weyl) anomaly:

$$
A_{C}:=\tau_{c}^{c}-\nabla_{\beta}\left(\varepsilon_{a b} \sigma^{a} e^{b \beta}\right)=-\left(a+\frac{\alpha_{3} p}{2}\right) \bar{e} R+\alpha_{3} \partial_{\beta}\left(\bar{e} \omega^{\beta}\right) .
$$

Here, the coefficient of $\bar{e} R$ is proportional to the sum of the central charges.

In treating the boundary symmetries of the MB model, Klemm et al. [8] followed a different approach, based on using the Riemannian connection in the renormalized action. Nevertheless, our results for anomalies coincide with theirs, in agreement with the theorem proved in section 3. The full strength of this theorem will be seen in the more interesting case of $3 \mathrm{D}$ gravity with propagating torsion, where the complicated field equations may lead to either vanishing or nonvanishing boundary torsion. However, we will be able to derive the Noether-Ward identities without recourse to the value of the boundary torsion.

\section{Holography in 3D gravity with propagating torsion}

In this section, we analyze the holographic structure of 3D gravity with propagating torsion, assuming parity invariance [12], and using the AdS asymptotic conditions (2.8). 


\subsection{Lagrangian and the field equations}

Assuming the absence of matter, dynamical content of 3D gravity with propagating torsion is defined by the action integral

$$
I=\int d^{3} x \hat{e} \mathcal{L}_{G},
$$

where $\hat{e}=\operatorname{det}\left(\hat{e}^{i}{ }_{\mu}\right)$, and the gravitational Lagrangian $\mathcal{L}_{G}$ is at most quadratic in the torsion and the curvature. Assuming parity invariance, the general form of $\mathcal{L}_{G}$ is given by [12]

$$
\mathcal{L}_{G}=-a \hat{R}-2 \Lambda_{0}+\mathcal{L}_{T^{2}}+\mathcal{L}_{R^{2}} .
$$

The quadratic terms can be conveniently be written in the form

$$
\begin{aligned}
& \mathcal{L}_{T^{2}}=\frac{1}{4} \hat{T}^{i j k} \mathcal{H}_{i j k}, \quad \mathcal{H}_{i j k}:=a_{1}{ }^{(1)} \hat{T}_{i j k}+a_{2}{ }^{(2)} \hat{T}_{i j k}+a_{3}{ }^{(3)} \hat{T}_{i j k}, \\
& \mathcal{L}_{R^{2}}=\frac{1}{8} \hat{R}^{i j k l} \mathcal{H}_{i j k l}, \quad \mathcal{H}_{i j k l}:=b_{4}{ }^{(1)} \hat{R}_{i j k l}+b_{5}{ }^{(2)} \hat{R}_{i j k l}+b_{6}{ }^{(3)} \hat{R}_{i j k l},
\end{aligned}
$$

where we introduced the covariant field momenta $\mathcal{H}_{i j k}$ and $\mathcal{H}_{i j k l}$, which are linear in the irreducible components of the torsion, ${ }^{(n)} \hat{T}_{i j k}$, and the curvature, ${ }^{(n)} \hat{R}_{i j k l}$. An equivalent form of these two terms, which is more convenient for practical calculations, is given by:

$$
\begin{aligned}
& \mathcal{H}_{i j k}=4\left(\alpha_{1} \hat{T}_{i j k}+\alpha_{2} \hat{T}_{[k j] i}+\alpha_{3} \hat{T}_{i j k}\right), \\
& \mathcal{L}_{R^{2}}=\hat{R}^{i j} \mathcal{H}_{i j}, \quad \mathcal{H}_{i j}=\beta_{1} \hat{R}_{i j}+\beta_{2} \hat{R}_{j i}+\beta_{3} \eta_{i j} \hat{R} .
\end{aligned}
$$

The expression for $\mathcal{L}_{R^{2}}$ is obtained using the fact that the Weyl tensor identically vanishes in $3 \mathrm{D}$, and the new coupling constants $\left(\alpha_{k}, \beta_{k}\right)$ are expressed in terms of the $\left(a_{k}, b_{k}\right)$ as [12]

$$
\begin{aligned}
\alpha_{1} & =\frac{1}{6}\left(2 a_{1}+a_{3}\right), \quad \alpha_{2}=\frac{1}{3}\left(a_{1}-a_{3}\right), \quad \alpha_{3}=\frac{1}{2}\left(a_{2}-a_{1}\right), \\
\beta_{1} & =\frac{1}{2}\left(b_{4}+b_{5}\right), \quad \beta_{2}=\frac{1}{2}\left(b_{4}-b_{5}\right), \quad \beta_{3}=\frac{1}{12}\left(b_{6}-4 b_{4}\right) .
\end{aligned}
$$

The variation of the action (5.1) with respect to $\hat{e}_{\mu}^{i}$ and $\hat{\omega}^{i j}{ }_{\mu}\left(=-\varepsilon^{i j} \hat{\omega}_{\mu}\right)$ produces two gravitational field equations, displayed in equations (2.13) of Ref. [12]. Without matter contribution, these equations, transformed to the local Lorentz basis, take the form:

$$
\begin{aligned}
& \nabla^{m} \mathcal{H}_{i m j}+\frac{1}{2} \mathcal{H}_{i}{ }^{m n}\left(-T_{j m n}+2 \eta_{j m} V_{n}\right)-t_{i j}=0, \\
& t_{i j}:=\eta_{i j} \mathcal{L}_{G}-T^{m n}{ }_{i} \mathcal{H}_{m n j}+2 a \hat{R}_{j i}-2\left(\hat{R}^{n}{ }_{i} \mathcal{H}_{n j}-\hat{R}_{j}{ }^{n m}{ }_{i} \mathcal{H}_{n m}\right),
\end{aligned}
$$

where $t_{i j}$ is the energy-momentum tensor of gravity, and

$$
2 a T_{k i j}+2 T^{m}{ }_{i j}\left(\mathcal{H}_{m k}-\eta_{m k} \mathcal{H}\right)+4 \nabla_{[i}\left(\mathcal{H}_{j] k}-\eta_{j] k} \mathcal{H}\right)+\varepsilon_{i j n} \varepsilon^{m r}{ }_{k} \mathcal{H}_{m r}{ }^{n}=0,
$$

with $\mathcal{H}=\mathcal{H}^{k}{ }_{k}$.

In the near-boundary expansion, the leading order of the field equations (5.3), corresponding to $\rho=0$, reduces to the following relations involving the coupling constants:

$$
\begin{aligned}
& p\left(a+q b_{6}+2 a_{3}\right)=0, \\
& a q-\Lambda_{0}+\frac{1}{2} p^{2} a_{3}-\frac{1}{2} q^{2} b_{6}=0 .
\end{aligned}
$$


As shown in [12], these relations ensure that the AdS configuration, as well as the black hole with torsion, are solutions of the present theory. However, quadratic equations (5.4) allow to have two different solution for the effective cosmological constant $\Lambda_{\text {eff }}=p-q^{2} / 4$, and consequently, two different AdS vacua. For a particular choice of parameters $(p=$ $\left.0, a-b_{6} q=0\right)$, the two vacua coincide [12]. For an analysis of this situation in the Bergshoeff-Hohm-Townsend gravity, see Refs. [27, 28].

\subsection{Equations of motion}

In this section, we discuss the consistency of the near-boundary analysis of the field equations (5.3), given in Appendix D, with the holographic description of the asymptotic theory.

The leading order of the field equations is given by Eqs. (5.4). These equations constrain the coupling constants and, therefore, restrict the form of the allowed gravity actions.

Equations linear in $\rho$ are given by the algebraic system (D.1), (D.2), (D.6) and (D.8) for the vector $\bar{V}_{a}=\bar{T}_{b a}^{b}$, which defines the complete torsion tensor in 2D (Appendix A). These equations allow not only Riemann-Cartan but also Riemann boundary geometries. However, thanks to the theorem proved in section 3, we can study the Noether-Ward identities in these two cases quite generally, without making an explicit distinction between them.

The order $\rho^{2}$ of the field equations is given in (D.3-D.5) and (D.7). These are algebraic equations in the tensor $s_{a b}$, which is related to the extrinsic curvature $K_{a b}$ (Appendix D). More precisely, these equations determine the antisymmetric part $\varepsilon^{a b} s_{a b}$ and the trace $s^{c}{ }_{c}$ as local expressions of the boundary curvature and torsion. In particular, for the vanishing torsion we have $\varepsilon^{a b} s_{a b}=0$ and $s^{c}{ }_{c}=\frac{1}{4} R$, as in the MB model.

Here, in contrast to the MB model, the radial expansion goes beyond $\rho^{2}$, but the cubic and higher order terms do not affect our results in the $\rho \rightarrow 0$ limit.

Let us emphasize that, in our near-boundary analysis, we were not able to determine the symmetric traceless part $s_{a b}^{\prime}$ of $s_{a b}$. We can understand this situation by noting that $s_{a b}^{\prime}$ is a nonlocal function that requires a global solution. Such nonlocal terms are parts of the (nonlocal) 1-point functions of the boundary CFT. On the other hand, physical objects, such as the conformal anomaly, are always local. This is a general feature of the boundary currents in an effective theory.

In the next section, we calculate the boundary currents of the effective CFT.

\subsection{Boundary currents}

In the absence of matter, the variation of the (gravitatonal) action, evaluated on-shell, takes the form

$$
\delta I_{\text {on-shell }}=\int d^{3} x \partial_{\mu}\left\{2 \varepsilon^{\mu \nu \lambda} \hat{e}_{\lambda}^{k}\left[\delta \hat{e}^{i}{ }_{\nu} \varepsilon^{j m}{ }_{k} \mathcal{H}_{i j m}+\delta \hat{\omega}^{i}{ }_{\nu}\left(a \eta_{i k}+\mathcal{H}_{k i}-\eta_{k i} \mathcal{H}\right)\right]\right\} .
$$

After expressing $\delta I_{\text {on-shell }}$ as a boundary integral, we will use the field equations to find the renormalized $2 \mathrm{D}$ action. Then, in accordance with $(3.5 \mathrm{~b})$, we will identify the energymomentum and the spin boundary currents as the objects (1-point functions) coupled to 
the sources $\bar{e}^{a}{ }_{\alpha}$ and $\bar{\omega}_{\alpha}$ in the boundary CFT. To do that, we write the action corresponding to the Lagrangian (5.2a) as

$$
I=I_{E C}+I_{\Lambda_{0}}+I_{T^{2}}+I_{R^{2}} .
$$

The variation of the term $I_{E C}$, linear in the scalar curvature, is known from the MB model:

$$
\begin{aligned}
\delta I_{E C}= & \frac{a p}{\rho^{2}} \int_{\partial \mathcal{M}} d^{2} x \varepsilon^{\alpha \beta} e^{a}{ }_{\alpha} \delta e_{a \beta}-4 a \int_{\partial \mathcal{M}} d^{2} x \varepsilon^{\alpha \beta} \varepsilon^{a b} s_{\alpha a} \delta e_{b \beta} \\
& +\delta \int_{\partial M} d^{2} x\left(\Delta_{1}+\Delta_{2}\right)
\end{aligned}
$$

where the total variation contains two pieces, one finite and the other divergent:

$$
\begin{aligned}
& \Delta_{1}:=4 a \varepsilon^{\alpha \beta} \varepsilon_{a b} s^{a}{ }_{\alpha} e^{b}{ }_{\beta}=-4 a e s^{c}{ }_{c}, \\
& \Delta_{2}:=-\frac{a}{\rho^{2}} \varepsilon^{\alpha \beta} \varepsilon_{a b} e^{a}{ }_{\alpha} e^{b}{ }_{\beta}=\frac{2 a}{\rho^{2}} \bar{e}\left(1+\rho^{2} s_{c}{ }_{c}\right) .
\end{aligned}
$$

The variation of the cosmological term does not contribute to the boundary integrals. Next, we vary the term quadratic in torsion:

$$
\delta I_{T^{2}}=\frac{2 a_{3} p}{\rho^{2}} \int_{\partial \mathcal{M}} d^{2} x \varepsilon^{\alpha \beta} e^{a}{ }_{\alpha} \delta e_{a \beta}+\frac{2 a_{3}}{\rho^{2}} \int_{\partial \mathcal{M}} d^{2} x(\hat{\mathcal{A}}-p) \varepsilon^{\alpha \beta} e^{a}{ }_{\alpha} \delta e_{a \beta} .
$$

Note that the second piece, containing the axial torsion, is a finite 2D integral.

Finally, the variation of the term quadratic in curvature yields:

$$
\begin{aligned}
\delta I_{R^{2}} & =2 \int_{\mathcal{M}} d^{3} x \varepsilon^{\mu \nu \sigma}\left(\mathcal{H}_{\sigma i}-b_{i \sigma} \mathcal{H}\right) \partial_{\mu} \delta \hat{\omega}^{i}{ }_{\nu} \\
& =2 \int_{\partial \mathcal{M}} d^{2} x \varepsilon^{\alpha \beta}\left[\mathcal{H}_{\alpha 1} \delta \omega_{\beta}+\left(\mathcal{H}_{c a}-\eta_{a c} \mathcal{H}\right) \frac{1}{\rho^{2}} e^{c}{ }_{\alpha} \delta k^{a}{ }_{\beta}\right],
\end{aligned}
$$

where

$$
\mathcal{H}_{c a}-\eta_{c a} \mathcal{H}=\eta_{c a} b_{6} q+\eta_{c a} \rho^{2}\left[b_{6} p(\varepsilon \cdot s)-\frac{b_{6}-b_{4}}{6}\left(R-4 s^{\gamma}{ }_{\gamma}\right)\right]+2 \varepsilon_{c a} \rho^{2} b_{5}(\varepsilon \cdot s),
$$

and $\varepsilon \cdot s:=\varepsilon^{f g} s_{f g}$. The first piece of $\delta I_{R^{2}}$ has the form

$$
A:=2 \beta_{2} \int_{\partial \mathcal{M}} d^{2} x \varepsilon^{\alpha \beta}\left(\frac{p}{2} \varepsilon_{a c}-\eta_{a c}\right) V^{c} \bar{e}^{a}{ }_{\alpha} \delta \omega_{\beta} .
$$

The second piece can be conveniently written as the sum of two terms, $B+C$, where:

$$
\begin{aligned}
B:= & \frac{b_{6} q p}{\rho^{2}} \int_{\partial \mathcal{M}} d^{2} x \varepsilon^{\alpha \beta} e_{a \alpha} \delta e^{a}{ }_{\beta}-4 b_{6} q \int_{\partial \mathcal{M}} d^{2} x \varepsilon^{\alpha \beta} \delta e_{a \alpha} \varepsilon^{a f} s_{\beta f} \\
& +\delta \int_{\partial M} d^{2} x \Delta_{3}, \\
\Delta_{3}:= & 4 b_{6} q \varepsilon^{\alpha \beta} \varepsilon^{a b} e_{a \alpha} s_{\beta b}-\frac{b_{6} q}{\rho^{2}} \varepsilon^{\alpha \beta} \varepsilon^{a b} e_{a \alpha} e_{b \beta}=\frac{q b_{6}}{\rho^{2}} \bar{e} K .
\end{aligned}
$$


and

$$
\begin{aligned}
C:= & 2 \int_{\partial \mathcal{M}} d^{2} x \varepsilon^{\alpha \beta}\left[b_{6} p(\varepsilon \cdot s)-\frac{b_{6}-b_{4}}{6}\left(R-4 s^{\gamma}{ }_{\gamma}\right)\right] e_{a \alpha}\left(\frac{p}{2} \eta^{a b}-\varepsilon^{a b}\right) \delta e_{b \beta} \\
& -4 b_{5} \int_{\partial \mathcal{M}} d^{2} x \varepsilon^{\alpha \beta}(\varepsilon \cdot s) e^{c}{ }_{\beta}\left(\frac{p}{2} \eta_{c a}-\varepsilon_{c a}\right) \delta e^{a}{ }_{\alpha} .
\end{aligned}
$$

Now, the first terms in $\delta I_{\mathrm{EC}}, \delta I_{\mathrm{T}^{2}}$ and $A$ are divergent, but their sum vanishes as a consequence of (5.4a). The sum $I_{\mathrm{ct}}:=\int d^{2} x\left(\Delta_{1}+\Delta_{2}+\Delta_{3}\right)$, which appears in $\delta I$ as a total variation and is also divergent, is recognized as the counterterm; when subtracted from $I$, it defines the renormalized action $I_{\text {ren }}=I-I_{\text {ct }}$, see the next subsection for more details. The variation of $I_{\text {ren }}$ is finite:

$$
\begin{aligned}
\delta I_{\text {ren }}= & -4 a \int_{\partial \mathcal{M}} d^{2} x \varepsilon^{\alpha \beta} \varepsilon^{a c} s_{\beta c} \delta e_{a \alpha}, \\
& +4 a_{3} \int_{\partial \mathcal{M}} d^{2} x(\varepsilon \cdot s) \varepsilon^{\alpha \beta} e^{a}{ }_{\alpha} \delta e_{a \beta}, \\
& +A-4 b_{6} q \int d^{2} x \varepsilon^{\alpha \beta} \varepsilon^{a f} s_{\beta f} \delta e_{a \alpha}+C .
\end{aligned}
$$

From this result, one can identify the spin and the energy-momentum boundary currents, or equivalently, the 1-point functions of an effective 2D quantum theory, as:

$$
\begin{aligned}
\sigma^{\beta}= & \left(b_{4}-b_{5}\right) \varepsilon^{\beta \alpha}\left(\frac{p}{2} \varepsilon_{a c}-\eta_{a c}\right) V^{c} \bar{e}^{a}{ }_{\alpha}, \\
\tau^{\alpha}{ }_{a}= & 4\left(a+b_{6} q\right) \varepsilon^{\alpha \beta} \varepsilon_{a c} s_{\beta}{ }^{c}+4 a_{3}(\varepsilon \cdot s) \varepsilon^{\alpha \beta} e_{a \beta} \\
& -\varepsilon^{\alpha \beta} \frac{b_{6}-b_{4}}{3}\left(R-4 s^{\gamma}{ }_{\gamma}\right) e^{b}{ }_{\beta}\left(\frac{p}{2} \eta_{b a}-\varepsilon_{b a}\right) \\
& +2 \varepsilon^{\alpha \beta}\left[\left(b_{6} \frac{p^{2}}{2}-2 b_{5}\right) \eta_{b a}+p\left(b_{5}-b_{6}\right) \varepsilon_{b a}\right] e^{b}{ }_{\beta}(\varepsilon \cdot s) .
\end{aligned}
$$

\subsection{Renormalized action}

Before we continue to examine the Noether-Ward identities of the boundary currents (5.12), let us stress that the variation of the full action $I$ contains the total variation of the divergent term $I_{\mathrm{ct}}$, which can be compactly expressed as

$$
I_{c t}=\frac{\left(a+q b_{6}\right)}{\rho^{2}} \int_{\partial M} d^{2} x \bar{e} K=\left(a+q b_{6}\right) \int_{\partial M} d^{2} x \tilde{e} K .
$$

Note that the factor $\left(a+q b_{6}\right)$ is proportional to the central charge of the theory [12]. Subtracting this counterterm from the original action $I$ yields the renormalized action,

$$
I_{\mathrm{ren}}=I-I_{c t}=I-\left(a+q b_{6}\right) \int_{\partial M} d^{2} x \tilde{e} K,
$$

the variation of which produces the finite boundary currents (5.12).

One should observe that here, like in the MB model or GR, the counterterm is of the Gibbons-Hawking type, but with a modified factor which involves the $\hat{R}^{2}$ coupling constant $b_{6}$. All the other quadratic terms in the action give finite contributions that 
need not be regularized. Similarly as in the previous section, we can decompose $I_{\text {ct }}$ into the Balasubramanian-Kraus type local counterterm and the finite term proportional to $\int d^{2} x \bar{e} s^{c}{ }_{c}$, which becomes, on shell, a local function of the boundary curvature and torsion.

We would like to emphasize that, in even boundary dimensions, there is a logarithmic term in the field expansions related to the variation of the conformal anomaly, i.e., to its functional derivative with respect to the corresponding source. In Einstein-Hilbert gravity, however, its coefficient is obtained as a variation with respect to the boundary metric of the conformal anomaly which is topological invariant in two dimensions, such that it can be dropped out in the holographic renormalization procedure [19]. For the present holographic analysis with torsion, the field equations can be also solved consistently without adding such type of terms. This seems to be a reflection of the fact that the coefficients of the log terms in both the vielbein and the spin connection are related to the variation of the Weyl anomaly that turns out to be, as we show below, a topological invariant, even when the torsional degrees of freedom are taken into account.

Similar type of a logarithmic term also appears in the action evaluated on-shell. Namely, the counterterm (5.13a) ensures a differentiable and finite variation of the action $I_{\text {ren }}$, but the action itself contains a log term whose coefficient is related to topological invariants. As mentioned in Sec.4, inclusion of these terms is important in the full renormalized action that is identified with the free energy of the dual CFT.

These invariants are the same as those appearing in the conformal anomaly, the form of which will be obtained in the next subsection.

\subsection{Boundary symmetries and anomalies}

To simplify the derivation of the boundary symmetries and make it more direct, we rewrite the spin and the energy-momentum current in a more compact way. First, using the expression (5.9), we write the spin current in the form

$$
\sigma^{\beta}=-2 \varepsilon^{\alpha \beta} \mathcal{H}_{\alpha 1}=\left.2 \varepsilon^{\beta \alpha}\left(\hat{e}^{a}{ }_{\alpha} \mathcal{H}_{a 1}\right)\right|_{\rho=0} .
$$

In what follows, we shall omit the sign $\left.\right|_{\rho=0}$ for simplicity. After isolating the counterterm, the energy-momentum tensor becomes

$$
\begin{aligned}
\tau_{b}^{\beta}= & 4\left(a+q b_{6}\right) \varepsilon^{\alpha \beta} \varepsilon_{c b} s_{\alpha}{ }^{c}-\frac{2 a_{3}}{\rho^{2}} \varepsilon^{\alpha \beta} e_{b \alpha}(\hat{\mathcal{A}}-p) \\
& -\frac{2}{\rho^{2}} \varepsilon^{\alpha \beta} e^{c}{ }_{\alpha}\left(\mathcal{H}_{c g}-\eta_{c g} \mathcal{H}-\eta_{c g} b_{6} q\right)\left(\frac{p}{2} \delta_{b}^{g}-\varepsilon^{g} b\right) .
\end{aligned}
$$

Then, using (5.4a), we obtain an equivalent form of $\tau^{\beta}{ }_{b}$ :

$$
\begin{aligned}
\tau_{b}^{\beta}= & -\frac{2}{\rho^{2}}\left(a+q b_{6}\right) \varepsilon^{\alpha \beta}\left(k_{b \alpha}-\varepsilon_{a b} e^{a}{ }_{\alpha}\right)-\frac{2 a_{3}}{\rho^{2}} \varepsilon^{\alpha \beta} e_{b \alpha} \hat{\mathcal{A}} \\
& -\frac{2}{\rho^{2}} \varepsilon^{\alpha \beta} e^{c}{ }_{\alpha}\left(\mathcal{H}_{c g}-\eta_{c g} \mathcal{H}-\eta_{c g} b_{6} q\right)\left(\frac{p}{2} \delta_{b}^{g}-\varepsilon^{g}{ }_{b}\right) .
\end{aligned}
$$

Note that the trace of $\tau^{\alpha}{ }_{a}$ is given by

$$
\tau=\bar{e}\left[-4\left(a+b_{6} q\right) s^{a}{ }_{a}-\frac{2}{\rho^{2}}\left(\mathcal{H}^{a}{ }_{a}-2 \mathcal{H}-2 b_{6} q+\frac{p}{2} \varepsilon^{a b} \mathcal{H}_{a b}\right)\right] .
$$


Lorentz invariance. To verify the conservation law of the spin current (5.14), we start from the relations:

$$
\begin{aligned}
\nabla_{\alpha} \sigma^{\alpha} & =\hat{\nabla}_{\alpha} \sigma^{\alpha}=\frac{\bar{e}}{\rho^{2}} \varepsilon^{b c}\left(\hat{T}^{a}{ }_{b c} \mathcal{H}_{a 1}+2 \hat{\nabla}_{b} \mathcal{H}_{c 1}\right) \\
\varepsilon^{a b} \tau_{a b} & =-\frac{2 \bar{e}}{\rho^{2}}\left(a+b_{6} q+2 a_{3}\right)(\hat{\mathcal{A}}-p)-\frac{2 \bar{e}}{\rho^{2}}\left(\varepsilon^{b c} \mathcal{H}_{b c}+\frac{p}{2} \mathcal{H}^{c}{ }_{c}-p \mathcal{H}-p b_{6} q\right) \\
& \equiv \frac{\bar{e}}{\rho^{2}}\left(a+2 a_{3}-\mathcal{H}^{c}{ }_{c}\right) \varepsilon^{a b} \hat{T}_{1 a b}=-\frac{2 \bar{e}}{\rho^{2}}\left(a+2 a_{3}-\mathcal{H}^{c}{ }_{c}\right) \hat{\mathcal{A}} .
\end{aligned}
$$

Then, using the field equation $(1 a b)$ in the form

$$
-2\left(a-\mathcal{H}^{c}{ }_{c}+2 a_{3}\right) \hat{\mathcal{A}}+\varepsilon^{b c}\left(\hat{T}^{a}{ }_{b c} \mathcal{H}_{a 1}+2 \hat{\nabla}_{b} \mathcal{H}_{c 1}\right)=0
$$

the Lorentz invariance condition is found to be satisfied on shell:

$$
A_{L} \equiv \nabla_{\alpha} \sigma^{\alpha}+\varepsilon^{a b} \tau_{a b}=0 .
$$

Thus, our parity-invariant model (5.2) is Lorentz-invariant, in contrast to the situation in the MB model, where the Chern-Simons term violates this invariance, see (4.12).

Translation invariance. Let us now examine the invariance under local translations. First, we note that the validity of the Lorentz invariance condition (3.6b) implies that the last term on the right-hand-side of (3.6a) vanishes. Next, we calculate the divergence of the energy-momentum tensor:

$$
\begin{aligned}
\nabla_{\beta} \tau^{\beta}{ }_{a}= & \frac{2 \bar{e}}{\rho^{2}}\left[\left(a+b_{6} q\right)\left(\frac{1}{\rho} \hat{R}_{1 a}-\left(\frac{p}{2} \varepsilon_{a b}-\eta_{a b}\right) V^{b}\right)+2 \nabla^{b} \mathcal{H}_{a 1 b}-a_{3}(p-\hat{\mathcal{A}}) \varepsilon_{a b} V^{b}\right] \\
& +\frac{\bar{e}}{\rho^{3}}\left(\varepsilon_{a}{ }^{b}+\frac{p}{2} \delta_{b}^{a}\right)\left[2 \varepsilon^{c d} \hat{\nabla}_{c}\left(\mathcal{H}_{d b}-\eta_{d b} \mathcal{H}\right)+2 \hat{\mathcal{A}} \mathcal{H}_{1 b}+2 \hat{\mathcal{A}} \mathcal{H}_{b 1}-2 k_{b}{ }^{d} \mathcal{H}_{d 1}\right. \\
& \left.+\hat{T}^{f}{ }_{c d} \varepsilon^{c d}\left(\mathcal{H}_{f b}-\eta_{f b} \mathcal{H}-b_{6} q \eta_{f b}\right)\right] .
\end{aligned}
$$

Making use of (5.4a) and the $(a b c)$ field equation (Appendix D), the above result is simplified:

$$
\begin{aligned}
\nabla_{\beta} \tau_{a}^{\beta}= & \frac{2 \bar{e}}{\rho^{2}}\left[\left(a+b_{6} q\right) \frac{1}{\rho} \hat{R}_{1 a}+2 \nabla^{b} \mathcal{H}_{a 1 b}-\alpha_{3}\left(\frac{p}{2} \varepsilon_{a b}+\eta_{a b}\right) V^{b}\right. \\
& \left.+a_{3}(\hat{\mathcal{A}}+p) \varepsilon_{a b} v^{b}+\frac{1}{\rho}\left(\varepsilon_{a b}+\frac{p}{2} \eta_{a b}\right)\left(\hat{\mathcal{A}} \mathcal{H}^{b}{ }_{1}-k^{d b} \mathcal{H}_{d 1}\right)\right]
\end{aligned}
$$

Then, using the relations

$$
\begin{aligned}
\sigma^{\beta} F_{\alpha \beta}= & \bar{e} R \mathcal{H}_{\alpha 1}, \\
\tau^{c b} T_{b a c}= & 4 \bar{e}\left(a+b_{6} q\right) s_{a b} V^{b}+\frac{2 \bar{e}}{\rho^{2}} a_{3}(p-\hat{\mathcal{A}}) \varepsilon_{a b} V^{b} \\
& -\frac{2 \bar{e}}{\rho^{3}}\left(\mathcal{H}_{a c}-\eta_{a c} \mathcal{H}-\eta_{a c} b_{6} q\right) \hat{R}_{1}{ }^{c},
\end{aligned}
$$


we finally obtain:

$$
\begin{aligned}
A_{T}= & \nabla_{\beta} \tau^{b}{ }_{a}-\sigma^{\beta} F_{a \beta}-\tau^{b c} T_{c a b} \\
= & \frac{\bar{e}}{\rho^{2}}\left[-4 \rho^{2}\left(a+b_{6} q+\alpha_{3}\right) s_{a b} V^{b}-\rho R \mathcal{H}_{a 1}+\frac{1}{\rho}\left(2 \hat{R}_{11}+\hat{R}\right) \mathcal{H}_{a 1}\right. \\
& \left.+\frac{2}{\rho}\left(\varepsilon_{a b}+\frac{p}{2} \eta_{a b}\right)\left(\hat{\mathcal{A}} \mathcal{H}^{b}{ }_{1}-k^{d b} \mathcal{H}_{d 1}\right)\right] \\
= & -4 \bar{e} s_{a}{ }^{b}\left[\left(a+b_{6} q+\alpha_{3}-\frac{b_{4}-b_{5}}{2}\left(1+\frac{p^{2}}{4}\right)\right) V_{b}+p \frac{b_{4}-b_{5}}{2} \varepsilon_{b c} V^{c}\right]=0,
\end{aligned}
$$

where, in the last line, we again used the $(a b c)$ field equation.

This proves the translation invariance on the boundary.

Conformal anomaly. Let us now examine the dilatation invariance by calculating the expression $A_{C}=\tau-\nabla_{\beta}\left(\varepsilon_{a b} \sigma^{a} e^{b \beta}\right)$. We start with

$$
\begin{aligned}
A_{C}= & \bar{e}\left[-4\left(a+b_{6} q\right) s_{c}^{c}+4 p\left(b_{5}-b_{6}\right)(\varepsilon \cdot s)+\frac{2}{3}\left(b_{6}-b_{4}\right)\left(R-4 s^{c}{ }_{c}\right)\right] \\
& +\left(b_{4}-b_{5}\right) \nabla_{\beta}\left[\bar{e}\left(\frac{p}{2} \varepsilon_{a b}-\eta_{a b}\right) e^{a \beta} V^{b}\right] .
\end{aligned}
$$

Then, the identity

$$
\nabla_{\beta}\left[\bar{e}\left(\frac{p}{2} \varepsilon_{a b}-\eta_{a b}\right) e^{a \beta} V^{b}\right]=\bar{e}\left[\left(\frac{p}{2} \varepsilon_{a b}-\eta_{a b}\right) \nabla^{a} V^{b}+V^{a} V_{a}\right],
$$

and the 2nd order piece of equation (1ab), lead to:

$$
\begin{aligned}
A_{C}= & \bar{e}\left[-\left(a+b_{6} q\right) R+4 b_{6} p q(\varepsilon \cdot s)+\left(a+q \frac{b_{6}+2 b_{4}}{3}\right)\left(R-4 s^{c}{ }_{c}\right)\right. \\
& \left.\left.-(q+2)\left(b_{4}-b_{5}\right)\left(\nabla_{a} V^{a}-V_{a} V^{a}\right)+p\left(b_{4}-b_{5}\right) \varepsilon^{a b} \nabla_{a} V_{b}\right)\right] .
\end{aligned}
$$

Finally, by using equations (1a) and (11), we obtain the conformal anomaly:

$$
\begin{aligned}
A_{C}= & -\left(a+b_{6} q\right) \bar{e} R+\left[2 \alpha_{3}-(q+2)\left(b_{4}-b_{5}\right)\right] \bar{e}\left(\nabla_{a} V^{a}-V_{a} V^{a}\right) \\
& +p\left(b_{4}-b_{5}\right) \bar{e} \varepsilon^{a b} \nabla_{a} V_{b} .
\end{aligned}
$$

Since the conformal symmetry is broken, the boundary symmetry is reduced to the local Poincaré invariance.

The first term in $A_{C}$, proportional to $\bar{e} R=\partial_{\alpha}\left(2 \varepsilon^{\alpha \beta} \omega_{\beta}\right)$, is a topological density (related to the topological invariant $\left.\int d^{2} x \bar{e} \bar{R}\right)$; the related factor $\left(a+b_{6} q\right)$ is proportional to the central charge of the theory [12]. Since the Weyl weights of $e^{a}{ }_{\alpha}, T^{a}{ }_{\beta \gamma}, V^{a}, \nabla_{a} V^{a}$ are $+1,+1,-1,-2$, respectively, the remaining two terms in $A_{C}$ are seen to be invariant under local dilatations. For details of the classification of conformal anomalies, see [29].

A closer inspection of the Weyl invariants leads to the identities:

$$
\begin{aligned}
& W_{1}:=\bar{e}\left(\nabla_{a} V^{a}-V_{a} V^{a}\right)=\partial_{\alpha}\left(\varepsilon^{\alpha \beta} e_{a \beta} \varepsilon^{a b} V_{b}\right), \\
& W_{2}:=\varepsilon^{a b} \nabla_{a} V_{b}=\partial_{\alpha}\left(\varepsilon^{\alpha \beta} e^{a}{ }_{\beta} V_{a}\right) .
\end{aligned}
$$


In particular, the first identity can be written in the language of differential forms as

$$
N^{\prime} \equiv T^{a *} T_{a}-e^{a} \nabla^{*} T_{a}=d\left(e^{a *} T_{a}\right),
$$

where we used $V_{a}=\varepsilon_{a b}{ }^{*} T^{b}$. The 2-form $N^{\prime}$, which represents $W_{1}$, has an interesting resemblance with the Nieh-Yan 4-form [30, 31]. Similarly, a Nie-Yan-like representation for $W_{2}$ is obtained by the replacement ${ }^{*} T_{a} \rightarrow T_{a}$ in (5.23). The integrals of $W_{1}$ and $W_{2}$ over the boundary are topological invariants, the nature of which will be studied elsewhere.

A theory with parameters for which the conformal anomaly vanishes is known as the critical gravity. For such a critical choice of parameters, the bulk theory may acquire logarithmic modes, which leads to a logarithmic CFT at the boundary. For general properties of gravities at the critical point, see e.g. [32].

\section{Concluding remarks}

In this paper, we presented an analysis of the AdS/CFT correspondence in the realm of $3 \mathrm{D}$ gravity with torsion, with an underlying RC geometry of spacetime.

Starting with a suitable holographic ansatz and its consistency condition, we found that the expected boundary symmetry is described by local Poincaré transformations plus dilatations. Based on an improved form of the Noether-Ward identities, we first analyzed the holographic features of the MB model, where we confirmed the results of Klemm and Tagliabue [8], derived by a different technique. Then, turning our attention to the more interesting case of 3D gravity with propagating torsion, we obtained the holographic conformal anomaly, with contributions stemming from both the curvature and the torsion invariants. As a consequence, the boundary symmetry is reduced to the local Poincaré invariance. The improved treatment of the Noether-Ward identities, being independent of the value of torsion on the boundary, significantly simplifies the calculations.

An interesting problem for further study is to clarify how torsion affects the structure of the dual CFT. A simple approach would be to study the specific PGT sectors containing only one of the six propagating torsion modes, with $J^{P}=0^{ \pm}, 1,2[12]$.

\section{Acknowledgments}

One of us (O.M.) would like to thank Max Bañados, Gastón Giribet, Julio Oliva, Jorge Zanelli and all the other participants of the Workshop on String Theory, Gravity and Fields, held in Buenos Aires in October 2012, for their useful comments in the final stage of the preparation of this manuscript. This work was supported by the Serbian Science Foundation under Grant No. 171031 and the Chilean FONDECYT Grants No. 1090357 and No. 1110102. O.M. thanks DII-PUCV for support through the project No. 123.711/2011. The work of R.O. is financed in part by the UNAB grant DI-117-12/R.

\section{A On the RC geometry in 2D}

In 2D, the Lorentz connection, which is Abelian, has only one independent "internal" component, $\omega^{a b}{ }_{\alpha}=-\varepsilon^{a b} \omega_{\alpha}$, and the local Poincaré transformations of $e^{a}{ }_{\alpha}$ and $\omega_{\alpha}$ have 
the form (2.12). The corresponding field strengths, the curvature and the torsion, are given by

$$
\begin{aligned}
& R^{a b}{ }_{\alpha \beta}=-\varepsilon^{a b} F_{\alpha \beta}, \quad F_{\alpha \beta}:=\partial_{\alpha} \omega_{\beta}-\partial_{\beta} \omega_{\alpha}, \\
& T^{a}{ }_{\alpha \beta}=\nabla_{\alpha} e^{a}{ }_{\beta}-\nabla_{\beta} e^{a}{ }_{\alpha}, \quad \nabla_{\alpha} e^{a}{ }_{\beta}:=\partial_{\alpha} e^{a}{ }_{\beta}-\varepsilon^{a}{ }_{c} \omega_{\alpha} e^{c}{ }_{\beta} .
\end{aligned}
$$

The Ricci tensor and the scalar curvature read:

$$
R_{c}^{a}=-\varepsilon^{a b} F_{c b}, \quad R=-\varepsilon^{a b} F_{a b} .
$$

As a consequence:

$$
R_{a b}=\frac{1}{2} \eta_{a b} R, \quad F_{a b}=\frac{1}{2} \varepsilon_{a b} R,
$$

and the Ricci tensor is always symmetric. The torsion tensor, with only two independent components, is completely determined by its vector piece $V_{a}=T_{b a}^{b}$ as

$$
T^{a}{ }_{b c}=\delta_{b}^{a} V_{c}-\delta_{c}^{a} V_{b} .
$$

When the torsion vanishes, the connection becomes Riemannian:

$$
\tilde{\omega}_{\alpha}=\frac{1}{2} \varepsilon^{a b}\left(c_{a b c}-c_{c a b}+c_{b c a}\right) e_{\alpha}^{c}, \quad c_{\alpha \beta}^{a}:=\partial_{\alpha} e^{a}{ }_{\beta}-\partial_{\beta} e^{a}{ }_{\alpha},
$$

see also (3.7).

In the Gauss-normal radial foliation, the unit normal to the boundary $\partial M$ has the form

$$
n_{i}=\left(n_{1}, n_{a}\right)=\frac{\hat{e}_{i}{ }^{\rho}}{\sqrt{-\hat{g}^{\rho \rho}}}=(1,0,0),
$$

with $n^{2}=-1$. The extrinsic curvature (the second fundamental form) of $\partial M$ is defined by $K_{i j}=\hat{\nabla}_{i} n_{j}$. The only nonvanishing components of $K_{i j}$ are

$$
K_{a b}:=\hat{\nabla}_{a} n_{b}=-\varepsilon_{b c} \hat{\omega}^{c}{ }_{a}=\varepsilon_{c b} k^{c}{ }_{a}=\frac{p}{2} \varepsilon_{a b}+\eta_{a b}-2 \rho^{2} s_{a b},
$$

where we used $k_{a}^{c}:=k_{\alpha}^{c} e_{a}^{\alpha}$. In particular:

$$
\begin{aligned}
& K_{(a b)}=\eta_{a b}-2 \rho^{2} s_{(a b)}, \quad K_{b}^{b}=2-2 \rho^{2} s^{c}{ }_{c}, \\
& \varepsilon^{a b} K_{a b}=-p-2 \rho^{2} \varepsilon^{a b} s_{a b} \equiv-\hat{\mathcal{A}} .
\end{aligned}
$$

The last equation gives an interesting geometric interpretation of the axial torsion $\hat{\mathcal{A}}$. For $\hat{\mathcal{A}}=0, K_{a b}$ reduces to the standard Riemannian form.

\section{B Residual symmetries to second order}

At the end of Section 2, we showed that the residual symmetry group with the parameter $f(x)$, defined by (2.9), acts as local dilatation on the leading order of the metric, $\bar{g}_{\alpha \beta}$. From (2.9), we can also find the transformation rule for the second order of the vielbein, $s^{a}{ }_{\alpha}$, and extend the result of Section 2 to the second order of the metric, $g_{(2) \alpha \beta}$. 
Indeed, using the definitions $\bar{g}_{\alpha \beta}=\eta_{a b} \bar{e}^{a}{ }_{\alpha} \bar{e}^{b}{ }_{\beta}$ and $g_{(2) \alpha \beta}=s_{\alpha \beta}+s_{\beta \alpha}$, and restricting our attention to dilatations $(f \neq 0)$, we obtain:

$$
\begin{aligned}
\delta_{f} \bar{g}_{\alpha \beta} & =2 f \bar{g}_{\alpha \beta}, \\
\delta_{f} g_{(2) \alpha \beta} & =2 f g_{(2) \alpha \beta}-2 \bar{e}_{a(\alpha} \bar{\nabla}_{\beta)} f^{a}+2 f^{\gamma} \bar{T}_{(\alpha \beta) \gamma},
\end{aligned}
$$

where $f_{\alpha}:=\frac{1}{2} \partial_{\alpha} f$. In the limit when torsion vanishes, this result reduces to the PenroseBrown-Henneaux transformation [33, 34], which was derived in Riemannian GR and used to study universal properties of trace anomalies.

\section{Field strengths and covariant momenta}

\section{C.1 Torsion and curvature}

The results of this subsection are obtained using the expression (2.11) for $k^{a b}$.

In the local Lorentz basis, the torsion components are:

$$
\begin{aligned}
& \hat{T}^{1}{ }_{1 c}=0 \\
& \hat{T}^{1}{ }_{b c}=\varepsilon_{c e} k_{b}^{e}-\varepsilon_{b e} k_{c}^{e}=-\varepsilon_{b c} k_{e}^{e}, \\
& \hat{T}^{a}{ }_{1 c}=-\varepsilon^{a e}\left[\varepsilon_{e c}+\left(\frac{p}{2} \eta_{e c}+k_{e c}\right)\right]+\rho e_{c}{ }^{\gamma} \partial_{\rho} e^{a}{ }_{\gamma}, \\
& \hat{T}^{a}{ }_{b c}=\rho T^{a}{ }_{b c},
\end{aligned}
$$

and the components of curvature read:

$$
\begin{aligned}
& \hat{R}_{11 c}=-\rho^{2} e_{c}^{\gamma} \partial_{\rho} \omega_{\gamma}, \\
& \hat{R}_{1 b c}=-\rho^{2} F_{b c}+\varepsilon^{e d} k_{e b} k_{d c}, \\
& \hat{R}_{a 1 c}=-\left(k_{a c}+\frac{p}{2} \varepsilon_{a}{ }^{b} k_{b c}\right)+\rho e_{c}{ }^{\gamma} \partial_{\rho} k_{a \gamma}, \\
& \hat{R}_{a b c}=\rho e_{b}{ }^{\beta} e_{c}{ }^{\gamma}\left(\nabla_{\beta} k_{a \gamma}-\nabla_{\gamma} k_{a \beta}\right) .
\end{aligned}
$$

The Ricci tensor and the scalar curvature are calculated from the relations:

$$
\begin{aligned}
& \hat{R}_{i k}=-\varepsilon^{m n}{ }_{i} \hat{R}_{m n k}, \\
& \hat{R}=-\varepsilon^{m n k} \hat{R}_{m n k}=\hat{R}^{1}{ }_{1}+\hat{R}^{a}{ }_{a} .
\end{aligned}
$$

Reduction. Equation (2.11), in which $k_{a b}$ is expressed in terms of $e^{a}{ }_{\alpha}$, simplifies the expressions (C.1) for the torsion:

$$
\begin{aligned}
& \hat{T}_{1 b c}=\varepsilon_{b c} \hat{\mathcal{A}}, \\
& \hat{T}_{a 1 c}=-\varepsilon_{a c} \hat{\mathcal{A}}, \\
& \hat{T}_{a b c}=\rho T_{a b c},
\end{aligned}
$$

where $\hat{\mathcal{A}}$ is the axial torsion:

$$
\hat{\mathcal{A}}:=\frac{1}{6} \varepsilon^{i j k} \hat{T}_{i j k}=p-\rho \varepsilon^{f g} e_{f}^{\beta} \partial_{\rho} e_{g \beta} .
$$


Similarly, the curvature tensor reads:

$$
\begin{aligned}
& \hat{R}_{11 c}=-\rho^{2} e_{c}^{\beta} \partial_{\rho} \omega_{\beta}, \\
& \hat{R}_{1 b c}=\varepsilon_{b c} q-\rho^{2} F_{b c}-\varepsilon_{b c} \frac{p}{2}(p-\hat{\mathcal{A}})+\rho \varepsilon_{b c} e^{g \beta} \partial_{\rho} e_{g \beta}+Y_{b c} \\
& \hat{R}_{a 1 c}=-\varepsilon_{a c} q+\left(\frac{p}{2} \varepsilon_{a c}-\eta_{a c}\right)(p-\hat{\mathcal{A}})+\rho^{3} \varepsilon_{a b} e^{b \beta} \partial_{\rho}\left(\rho^{-1} \partial_{\rho} e_{c \beta}\right)+X_{a c} \\
& \hat{R}_{a b c}=\rho\left(\frac{p}{2} T_{a b c}-\varepsilon_{a}{ }^{f} T_{f b c}\right)+Z_{a b c}
\end{aligned}
$$

where $Y_{a c}, X_{a c}$ and $Z_{a b c}$ are given by

$$
\begin{aligned}
& X_{a c}:=\rho^{2} \varepsilon_{a}{ }^{f} e_{c}{ }^{\beta} \partial_{\rho} e_{b \gamma} \partial_{\rho}\left(e_{f}^{\gamma} e^{b}{ }_{\beta}\right)=-\eta_{a c} \rho^{3} \partial_{\rho}\left[\rho^{-2}(p-\hat{\mathcal{A}})\right]-\rho(p-\hat{\mathcal{A}}) e_{c}{ }^{\beta} \partial_{\rho} e_{a \beta}, \\
& Y_{b c}=-\rho^{2} \varepsilon_{f g} e^{f \beta} e^{g \gamma}\left(\partial_{\rho} e_{b \beta}\right)\left(\partial_{\rho} e_{c \gamma}\right), \\
& Z_{a b c}=-\rho^{2} \varepsilon_{a f} e_{b}{ }^{\beta} e_{c}^{\gamma}\left[\nabla_{\beta}\left(\partial_{\rho} e^{f \alpha} g_{\alpha \gamma}\right)-\nabla_{\gamma}\left(\partial_{\rho} e^{f \alpha} g_{\alpha \beta}\right)\right] .
\end{aligned}
$$

As a consequence, the Ricci tensor and the scalar curvature read:

$$
\begin{aligned}
\hat{R}_{11}= & \varepsilon^{b c} \hat{R}_{b 1 c}=2 q-p(p-\hat{\mathcal{A}})-\rho^{3} e^{c \beta} \partial_{\rho}\left(\rho^{-1} \partial_{\rho} e_{c \beta}\right)+(p-\hat{\mathcal{A}})^{2}, \\
\hat{R}_{1 c}= & -\varepsilon^{a b} \hat{R}_{a b c}=\rho\left(\frac{p}{2} \varepsilon_{c b} V^{b}-V_{c}\right)-\varepsilon^{a b} Z_{a b c}, \\
\hat{R}_{a 1}= & \varepsilon_{a}{ }^{c} \hat{R}_{11 c}=-\rho^{2} \varepsilon_{a}{ }^{c} e_{c}{ }^{\beta} \partial_{\rho} \omega_{\beta}, \\
\hat{R}_{a b}= & \varepsilon_{a}{ }^{c}\left(\hat{R}_{c 1 b}-\hat{R}_{1 c b}\right)=-2 \eta_{a b} q+\left(p \eta_{a b}-\varepsilon_{a b}\right)(p-\hat{\mathcal{A}}) \\
& +\rho^{3} e_{a}^{\beta} \partial_{\rho}\left(\rho^{-1} \partial_{\rho} e_{b \beta}\right)+\rho^{2} R_{a b}-\rho \eta_{a b} e^{g \gamma} \partial_{\rho} e_{g \gamma}+\varepsilon_{a}^{c}\left(X_{c b}-Y_{c b}\right), \\
\hat{R}=- & 6 q+3 p(p-\hat{\mathcal{A}})+2 \rho^{3} e^{c \beta} \partial_{\rho}\left(\rho^{-1} \partial_{\rho} e_{c \beta}\right) \\
& +\rho^{2} R-2 \rho e^{f \beta} \partial_{\rho} e_{f \beta}-\varepsilon^{a c}\left(2 X_{a c}+Y_{a c}\right),
\end{aligned}
$$

where

$$
\begin{gathered}
\varepsilon_{a}^{c}\left(X_{c b}-Y_{c b}\right)=-\varepsilon_{a b} \rho^{3} \partial_{\rho}\left[\rho^{-2}(p-\hat{\mathcal{A}})\right]-\rho(p-\hat{\mathcal{A}}) \varepsilon_{a}{ }^{c} e_{b}{ }^{\beta} \partial_{\rho} e_{c \beta} \\
+\rho^{2} \varepsilon_{a}{ }^{c} E^{\beta \gamma}\left(\partial_{\rho} e_{c \beta}\right)\left(\partial_{\rho} e_{b \gamma}\right) \\
-\varepsilon^{a c}\left(2 X_{a c}+Y_{a c}\right)=-2(p-\hat{\mathcal{A}})^{2}-\rho^{2} \varepsilon^{b c} E^{\beta \gamma}\left(\partial_{\rho} e_{b \beta}\right)\left(\partial_{\rho} e_{c \gamma}\right) .
\end{gathered}
$$

\section{C.2 Covariant momenta}

Here, we rely on the conditions (2.8), which imply $X_{a b c}=\mathcal{O}_{4}=Y_{a b c}$ and $Z_{a b c}=\mathcal{O}_{3}$. The calculations in section 5 are greatly simplified if we first find the explicit form of the covariant momenta. In the torsion sector, we have:

$$
\begin{aligned}
& \mathcal{H}_{11 c}=-2 \alpha_{3} \rho V_{c}, \\
& \mathcal{H}_{1 b c}=-\mathcal{H}_{b 1 c}=4\left(\alpha_{1}-\alpha_{2}\right) \varepsilon_{b c} \hat{\mathcal{A}}, \\
& \mathcal{H}_{a b c}=2 \rho\left(2 \alpha_{1}+\alpha_{2}+\alpha_{3}\right) T_{a b c},
\end{aligned}
$$


and in the curvature sector, we find:

$$
\begin{aligned}
\mathcal{H}_{11}= & 2\left(\beta_{1}+\beta_{2}+3 \beta_{3}\right) q-\left(\beta_{1}+\beta_{2}+3 \beta_{3}\right) p(p-\hat{\mathcal{A}}) \\
& -\beta_{3} \rho^{2}\left(R-4 s^{c}{ }_{c}\right)+\mathcal{O}_{4}, \\
\mathcal{H}_{a 1}= & \beta_{2} \rho\left(\frac{p}{2} \varepsilon_{a c}-\eta_{a c}\right) V^{c}+\mathcal{O}_{3} \\
\mathcal{H}_{1 a}= & \beta_{1} \rho\left(\frac{p}{2} \varepsilon_{a c}-\eta_{a c}\right) V^{c}+\mathcal{O}_{3}, \\
\mathcal{H}_{a b}= & -2\left(\beta_{1}+\beta_{2}+3 \beta_{3}\right) \eta_{a b} q+\left(\beta_{1}+\beta_{2}+3 \beta_{3}\right) \eta_{a b} p(p-\hat{\mathcal{A}}) \\
& -\left(\beta_{1}-\beta_{2}\right) \varepsilon_{a b}(p-\hat{\mathcal{A}})+\frac{1}{2} \rho^{2}\left(\beta_{1}+\beta_{2}+2 \beta_{3}\right) \eta_{a b}\left(R-4 \bar{e}^{f \beta} s_{f \beta}\right)+\mathcal{O}_{4} .
\end{aligned}
$$

\section{Radial expansion of the field equations}

In this Appendix, we display higher orders in $\rho$ of the vacuum field equations (5.3), which are needed in our study of the Noether-Ward identities for 3D gravity with propagating torsion. To zeroth order in $\rho$, the content of these equations is displayed in (5.4). The parameter $q$ is given in $(2.14 \mathrm{~b})$ as $q=p^{2} / 4-1$. In our notation, $\varepsilon \cdot s=\varepsilon^{a b} s_{a b}$ and $\mathcal{H}=\mathcal{H}^{k}{ }_{k}$.

(1) Let us start by considering the components $(i j)=(1 a),(a 1),(11)$ and $(a b)$ of the first field equation (5.3a). The object $t_{i j}$ is defined in the same equation. For each component $(i, j)$, we display first a compact form, and then the fully expanded field equation.

$(1 a)$ :

$$
\begin{aligned}
& \hat{\nabla}^{m} \mathcal{H}_{1 a m}+\frac{1}{2} \mathcal{H}_{1}{ }^{m n} \hat{T}_{a m n}-\mathcal{H}_{1 a}{ }^{n} V_{n}+t_{1 a}=0, \\
& 2 \rho\left[\left(2 \alpha_{1}+\alpha_{2}+\alpha_{3}\right)+\beta_{1} q\right]\left(\frac{p}{2} \varepsilon_{a b} V^{b}-V_{a}\right)=\mathcal{O}_{3} .
\end{aligned}
$$

$(a 1)$ :

$$
\begin{aligned}
& \hat{\nabla}^{m} \mathcal{H}_{a 1 m}+\frac{1}{2} \mathcal{H}_{a}{ }^{m n} \hat{T}_{1 m n}-\mathcal{H}_{a 1}{ }^{n} V_{n}+t_{a 1}=0, \\
& -2 \rho\left[a+\alpha_{3}+\left(b_{6}+\beta_{2}\right) q\right] V_{a} \\
& +p \rho\left[a-\alpha_{3}+8\left(\alpha_{1}-\alpha_{2}\right)+\left(b_{6}+\beta_{2}\right) q\right] \varepsilon_{a b} V^{b}=\mathcal{O}_{3} .
\end{aligned}
$$

(11):

$$
\begin{aligned}
& \hat{\nabla}^{m} \mathcal{H}_{11 m}+\frac{1}{2} \mathcal{H}_{1}{ }^{m n} \hat{T}_{1 m n}-\mathcal{H}_{11}{ }^{n} V_{n}+t_{11}=0, \\
& -2 \alpha_{3} \nabla_{a} V^{a}-\left[\left(2 \alpha_{1}+\alpha_{2}-\alpha_{3}\right)+\beta_{1} q\right] V_{c} V^{c} \\
& +\left[a-\left(2 \beta_{3}-\frac{b_{6}}{2}\right) q\right]\left(R-4 s^{\gamma}{ }_{\gamma}\right) \\
& -2 p\left[a+4\left(\alpha_{1}-\alpha_{2}\right)-b_{6} q\right](\varepsilon \cdot s)=\mathcal{O}_{2} .
\end{aligned}
$$


$(a b)$ :

$$
\begin{aligned}
& \hat{\nabla}^{m} \mathcal{H}_{a b m}+\frac{1}{2} \mathcal{H}_{a}{ }^{m n} \hat{T}_{b m n}-\mathcal{H}_{a b}{ }^{n} V_{n}+t_{a b}=0, \\
& 2\left(2 \alpha_{1}+\alpha_{2}+\alpha_{3}\right) \nabla^{c} T_{a b c}-\left[\left(2 \alpha_{1}+\alpha_{2}+\alpha_{3}\right)+\beta_{1} q\right] \eta_{a b} V_{c} V^{c} \\
& -\eta_{a b}\left(\beta_{3}-\frac{3 b_{6}}{4}\right) q\left(R-4 s^{\gamma} \gamma\right) \\
& +2 \eta_{a b}\left[a+4\left(\alpha_{1}-\alpha_{2}\right)-b_{6} q\right] p(\varepsilon \cdot s) \\
& -4 \varepsilon_{a b}\left[a+4\left(\alpha_{1}-\alpha_{2}\right)-\left(\beta_{1}-\beta_{2}-b_{6}\right) q\right](\varepsilon \cdot s)=\mathcal{O}_{2} .
\end{aligned}
$$

(2) Now, we turn to the components $(k i j)=(a 1 b),(11 b),(1 a b)$ and $(c a b)$ of the second field equation (5.3b).

$(a 1 b)$ :

$$
\begin{aligned}
& 2 \hat{T}^{c}{ }_{1 b}\left(a \eta_{c a}+\mathcal{H}_{c a}-\eta_{c a} \mathcal{H}\right)+2 \hat{\nabla}_{1}\left(\mathcal{H}_{b a}-\eta_{b a} \mathcal{H}\right)-2 \hat{\nabla}_{b} \mathcal{H}_{1 a}-\varepsilon_{b c} \varepsilon^{f}{ }_{a}\left(\mathcal{H}_{1 f}{ }^{c}-\mathcal{H}_{f 1}{ }^{c}\right)=0, \\
& -2 \beta_{1}\left(\frac{p}{2} \varepsilon_{a f}-\eta_{a f}\right) \nabla_{b} V^{f}+2 p \eta_{a b}\left[2 b_{6}+\left(\beta_{1}-\beta_{2}\right)\right](\varepsilon \cdot s) \\
& -4 \varepsilon_{a b}\left[a+4\left(\alpha_{1}-\alpha_{2}\right)+b_{6}\left(q+p^{2} / 2\right)+\left(\beta_{1}-\beta_{2}\right)\right](\varepsilon \cdot s) \\
& -\left(\eta_{a b}-\frac{p}{2} \varepsilon_{a b}\right)\left(\frac{3 b_{6}}{4}-\beta_{3}\right)\left(R-4 s^{\gamma}{ }_{\gamma}\right)=\mathcal{O}_{2} .
\end{aligned}
$$

$(11 b)$ :

$$
\begin{aligned}
& 2 \hat{T}^{c}{ }_{1 b} \mathcal{H}_{c 1}+2 \hat{\nabla}_{1} \mathcal{H}_{b 1}-2 \hat{\nabla}_{b}\left(\mathcal{H}_{11}-\eta_{11} \mathcal{H}^{k}{ }_{k}\right)+\varepsilon_{b c} \varepsilon^{f g} \mathcal{H}_{f g}{ }^{c}=0, \\
& {\left[-\left(2 \alpha_{1}+\alpha_{2}+\alpha_{3}\right)-\beta_{1} q\right] \rho V_{b}=\mathcal{O}_{3} .}
\end{aligned}
$$

$(1 a b)$ :

$$
\begin{aligned}
& 2 \hat{T}^{1}{ }_{a b}\left(a \eta_{11}+\mathcal{H}_{11}-\eta_{11} \mathcal{H}\right)+2 \hat{T}^{c}{ }_{a b} \mathcal{H}_{c 1}+4 \hat{\nabla}_{[a} \mathcal{H}_{b] 1}+\varepsilon_{a b} \varepsilon^{f g} \mathcal{H}_{f g}{ }^{1}=0, \\
& 4\left[a+4\left(\alpha_{1}-\alpha_{2}\right)+b_{6}\left(q+p^{2} / 2\right)-2\left(\beta_{1}-\beta_{2}\right)\right](\varepsilon \cdot s) \\
& +p \beta_{2} V_{c} V^{c}-2 \beta_{2}\left(\frac{p}{2} \eta^{f g}-\varepsilon^{f g}\right) \nabla_{f} V_{g}-p\left(\frac{b_{6}}{4}+\beta_{3}\right)\left(R-4 s_{\gamma}^{\gamma}\right)=\mathcal{O}_{2} .
\end{aligned}
$$

$(c a b)$ :

$$
\begin{aligned}
& 2 \hat{T}^{f}{ }_{a b}\left(a \eta_{f c}+\mathcal{H}_{f c}-\eta_{f c} \mathcal{H}\right)+2 \hat{T}^{1}{ }_{a b} \mathcal{H}_{1 c}+4 \hat{\nabla}_{[a}\left(\mathcal{H}_{b] c}-\eta_{b] c} \mathcal{H}\right)-\varepsilon_{a b} \varepsilon^{f} \mathcal{H}_{1 f}{ }^{1}=0, \\
& {\left[a+b_{6} q-\beta_{2}\left(1+\frac{p^{2}}{4}\right)+\alpha_{3}\right] T_{c a b}-\beta_{2} p \varepsilon_{a b} V_{c}=\mathcal{O}_{2} .}
\end{aligned}
$$

\section{References}

[1] E. Witten, Anti-de Sitter space and holography, Adv. Theor. Math. Phys. 2 (1998) 253.

[2] O. Aharony, S. S. Gubser, J. Maldacena, H. Ooguri and Y. Oz (2000), Large N field theories, string theory and gravity, Physics Reports 323 (2000) 183. 
[3] R. Bousso, The Holographic principle, Rev. Mod. Phys. 74 (2002) 825.

[4] M. Blagojević, Gravitation and gauge symmetries, IoP Publishing, Bristol (2002).

[5] T. Ortín, Gravity and strings, Cambridge University Press, Cambridge (2004).

[6] M. Blagojević and F. W. Hehl, Gauge Theories of Gravitation-A Reader with Commentaries, Imperial College Press, London (2013).

[7] M. Bañados, O. Mišković, and S. Theisen, Holographic currents in first order gravity and finite Fefferman-Graham expansions, JHEP 06 (2006) 025.

[8] D. Klemm and G. Tagliabue, The CFT dual of AdS gravity with torsion, Class. Quantum Grav. 25 (2008) 035011.

[9] E. W. Mielke and P. Baekler, Topological gauge model of gravity with torsion, Phys. Lett. A 156 (1991) 399.

[10] A. C. Petkou, Torsional degrees of freedom in $A d S_{4} / C F T_{3}$ [arXiv:1004.1640 [hep-th]].

[11] M. Blagojević and B. Cvetković, Canonical structure of 3D gravity with torsion, in: Progress in General Relativity and Quantum Cosmology, vol. 2, ed. Ch. Benton, Nova Science Publishers, New York (2006), pg. 103 [gr-qc/0412134].

[12] M. Blagojević and B. Cvetković, 3D gravity with propagating torsion: the AdS sector, Phys. Rev. D 85 (2012) 104003.

[13] J. A. Helayël-Neto, C. A. Hernaski, B. Pereira-Dias, A. A. Vargas-Paredes, and V. J. Vasquez-Otoya, Chern-Simons gravity with (curvature) $)^{2}$ - and (torsion) $)^{2}$-terms and a basis of degree-of-freedom projection operators, Phys. Rev. D 82 (2010) 064014.

[14] S. Deser, R. Jackiw and S. Templeton, Three-dimensional massive gauge theories, Phys. Rev. Lett. 48 (1982) 975.

[15] S. Deser, R. Jackiw and S. Templeton, Topologically massive gauge theories, Ann. Phys. 140 (1982) 372 .

[16] E. A. Bergshoeff, O. Hohm and P. K. Townsend, Massive gravity in three dimensions, Phys. Rev. Lett. 102 (2009) 201301.

[17] P. A. M. Dirac, Forms of relativistic dynamics, Rev. Mod. Phys. 21 (1949) 392.

[18] C. Fefferman and R. Graham, Conformal invariants, in: The mathematical heritage of Elie Cartan, Lyon 1984, Astérisque, 1985, Numero Hors Serie, 95.

[19] K. Skenderis, M. Taylor and B. C. van Rees, Topologically Massive Gravity and the AdS/CFT Correspondence, JHEP 0909 (2009) 045.

[20] J. D. Brown and M. Henneaux, Central charges in the canonical realization of asymptotic symmetries: An example from three dimensional gravity, Comm. Math. Phys. 104 (1986) 207.

[21] M. Blagojević and B. Cvetković, Canonical structure of topologically massive gravity with a cosmological constant, JHEP 0905 (2009) 073.

[22] K. Skenderis and S. N. Solodukhin, Quantum effective action from the AdS/CFT correspondence, Phys. Lett. B 472 (2000) 316.

[23] M. Bañados and F. Méndez, Note on covariant action integrals in three dimensions, Phys. Rev. D 58 (1998) 104014. 
[24] O. Mišković and R. Olea, On boundary conditions in three-dimensional AdS gravity, Phys. Lett. B 640 (2006) 101.

[25] R. Olea, Regularization of odd-dimensional AdS gravity: Kounterterms, JHEP 0704 (2007) 073.

[26] V. Balasubramanian and P. Kraus, A Stress tensor for Anti-de Sitter gravity, Commun. Math. Phys. 208 (1999) 413.

[27] G. Giribet and M. Leston, Boundary stress tensor and counterterms for weakened AdS3 asymptotic in New Massive Gravity, JHEP 1009 (2010) 070.

[28] G. Giribet, J. Oliva, D. Tempo and R. Troncoso, Microscopic entropy of the three-dimensional rotating black hole of BHT massive gravity, Phys. Rev. D 80 (2009) 124046 .

[29] S. Deser and A. Schwimmer, Geometric classification of conformal anomalies in arbitrary dimensions, Phys. Lett. B 309 (1993) 279.

[30] H. T. Nieh and M. L. Yan, An identity in Riemann-Cartan geometry, J. Math. Phys. 23 (1982) 373.

[31] H. T. Nieh, A torsional topological invariant, Int. J. Mod. Phys. A 22 (2007) 5237.

[32] S. Deser, H. Liu, H. Lu, C. N. Pope, T. C. Sisman and B. Tekin, Critical points of D-dimensional extended gravities, Phys. Rev. D 83 (2011) 061502.

[33] C. Imbimbo, A. Schwimmer, S. Theisen and S. Yankielowicz, Diffeomorphisms and holographic anomalies, Class. Quant. Grav. 17 (2000) 1129.

[34] A. Schwimmer and S. Theisen, Universal features of holographic anomalies, JHEP 0310 (2003) 001. 